Daniel Aubram, Stavros A. Savidis, Frank Rackwitz

\title{
Theory and Numerical Modeling of Geomechanical Multi-material Flow
}

Chapter in book | Accepted manuscript (Postprint)

This version is available at https://doi.org/10.14279/depositonce-9150

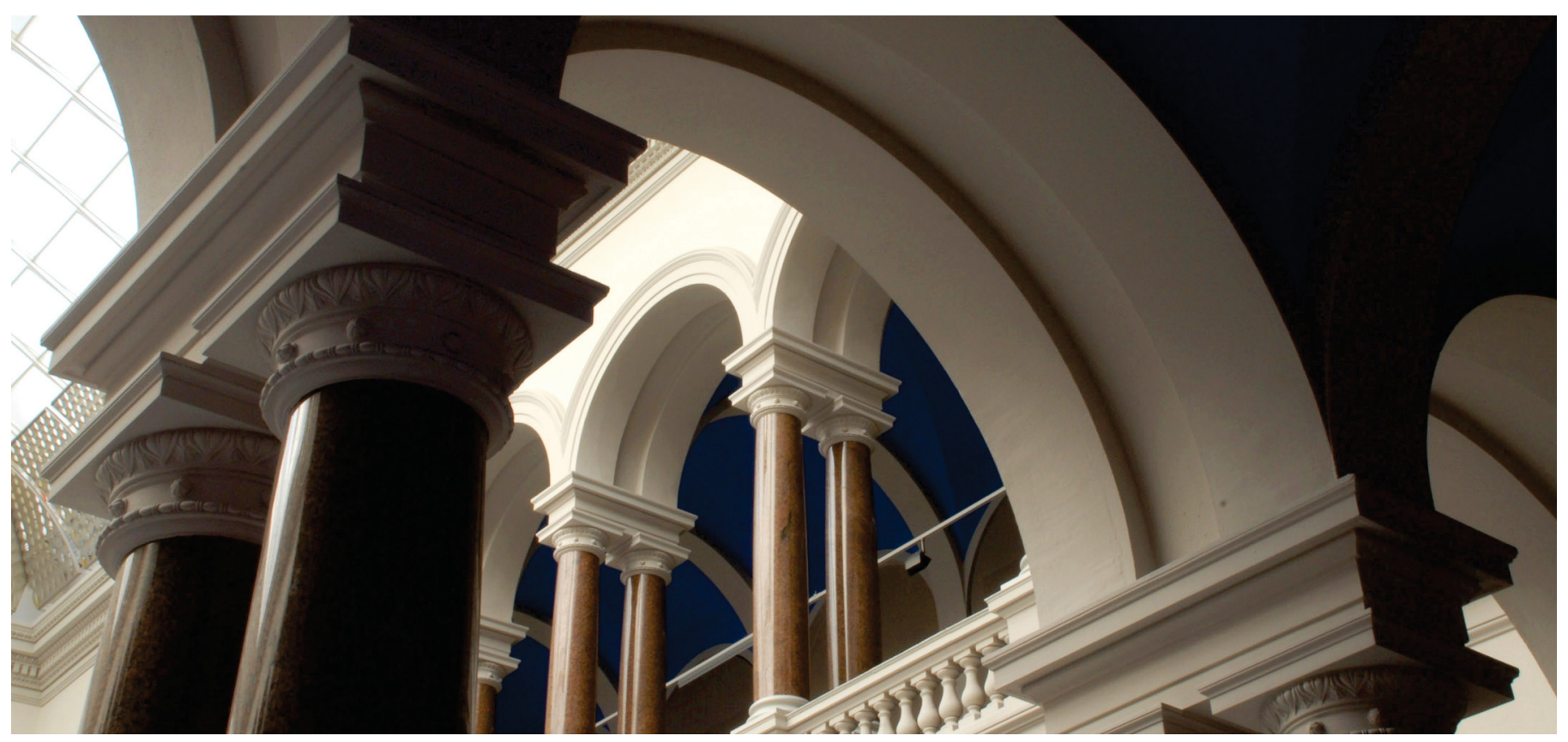

Aubram, D., Savidis, S. A., \& Rackwitz, F. (2015). Theory and Numerical Modeling of Geomechanical Multi-material Flow. In Holistic Simulation of Geotechnical Installation Processes (pp. 187-229). Springer International Publishing. https://doi.org/10.1007/978-3-319-23159-4_10 


\title{
Theory and Numerical Modeling of Geomechanical Multi-Material Flow
}

\author{
Daniel Aubram ${ }^{1}$, Stavros A. Savidis ${ }^{1}$, and Frank Rackwitz ${ }^{2}$ \\ 1 Chair of Soil Mechanics and Geotechnical Engineering, Berlin University of \\ Technology, Secr. TIB1-B7, Gustav-Meyer-Allee 25, D-13355 Berlin, Germany \\ 2 Department of Geotechnical Engineering, Ostbayerische Technische Hochschule \\ Regensburg, Prüfeninger Straße 58, D-93049 Regensburg, Germany
}

\begin{abstract}
Multi-material flow describes a situation where several distinct materials separated by sharp material interfaces undergo large deformations. The research presented in this paper addresses a particular class of multi-material flow situations encountered in geomechanics and geotechnical engineering which is characterized by a complex coupled behavior of saturated granular material as well as by a hierarchy of distinct spatial scales. Examples include geotechnical installation processes, liquefaction-induced soil failure, and debris flow. The most attractive numerical approaches to solve such problems use variants of arbitrary Lagrangian-Eulerian descriptions allowing interfaces and free surfaces to flow through the computational mesh. Mesh elements cut by interfaces (multi-material elements) necessarily arise which contain a heterogeneous mixture of two or more materials. The heterogeneous mixture is represented as an effective single-phase material by using mixture theory. The paper outlines the specific three-scale mixture theory developed by the authors and the MMALE numerical method to model and simulate geomechanical multi-material flow. In contrast to traditional flow models which consider the motion of multiple single-phase materials or single multi-phase mixtures, the present research succeeds in incorporating both the coupled behavior of saturated granular material and its interaction with other (pure) materials.
\end{abstract}

Keywords: multi-material flow, large deformations, mixture, granular material, sand, volume averaging, interface reconstruction, volume of fluid method

\section{Introduction}

Geotechnical installation processes which may include digging, mixing, displacement, or penetration, are characterized by large material deformations, moving material interfaces and free surfaces, changing contact conditions, and complex nonlinear soil mechanical behavior [112]. Soil generally is a mixture of solid grains and one or more pore fluids (liquid and/or gas). Its mechanical behavior results from the behavior of each constituent, the internal structure, and from the interfacial coupling due to mass and momentum transfer. The realistic simulation of

NOTICE: This is the author's version of a work that was accepted for publication. A definitive version of this article is published in:

D. Aubram, S. A. Savidis, F. Rackwitz. Theory and Numerical Modeling of Geomechanical Multi-Material Flow. In Th. Triantyfyllidis (ed): Holistic Simulation of Geotechnical Installation Processes - Benchmarks and Simulations, pages 187-229. Springer International Publishing, 2016. http://dx.doi.org/10.1007/978-3-319-23159-4_10 
such complex processes, either numerically or by experiments, is very challenging but at the same time of high practical relevance because geotechnical installation processes may significantly impact on the soil and the load bearing of nearby structures. This is particularly the case for the installation of vibro-injection piles [84,92], which has recently been investigated by the authors [14,98]; see also Fig. 1a.

There are several more situations encountered in geomechanics and geotechnical engineering that share the characteristics of geotechnical installation processes. Examples are liquefaction-induced soil failure $[104,105]$, natural hazards like landslides interacting with water [73,78], and debris flow $[63,64]$. Schematic views are shown in Fig. 1. To make their similarities clear, consider the liquefaction-induced failure of an earth-fill dam under seismic excitation (Fig. 1b). Usually the details of the exact flow fields (e.g. motion of particular grains) are of secondary interest. However, the consideration of the liquefactionprone, water-saturated fill material as a mixture of granular material and pore fluid is indispensable [120]. At the same time the instantaneous water level and geometry of the dam (free surfaces and large-scale interfaces) must be taken into account because they govern the progress of failure. The initiation and evolution of the liquefied zone is generally unknown and could only be resolved in a direct numerical simulation of the problem. In this regard major achievements have been made by using Lagrangian or almost-Lagrangian descriptions in which mesh elements contain only one material throughout the calculation $[81,120,121]$. However, a simulation of the entire process from flow initiation to deposit consolidation will fail due to severe mesh distortion unless more sophisticated approaches will be employed.

We refer to each of the situations mentioned above as geomechanical multimaterial flow. Multi-material flow generally contains several pure, physically distinct materials which are separated by sharp material interfaces and one or more of these materials undergo large deformations - void (empty space or atmosphere) is considered as material. In contrast to traditional multi-phase or multi-fluid flow, material strength and compressibility should be included in the description of multi-material flow, whereas mass transfer between the materials is usually of secondary interest. Moreover, in many situations momentum relaxation can be assumed infinitely fast, resulting in a velocity field common to all materials in the flow.

The notion of multi-material flow has emerged along with the development of efficient numerical simulation techniques $[24,75]$. Problems that have traditionally been modeled include hypervelocity impact, detonation with structuremedia interaction, dynamics of bubbles and droplets, material processing and manufacturing, as well as astrophysical events. The most attractive approaches use variants of the multi-material arbitrary Lagrangian-Eulerian (MMALE) description allowing interfaces and free surfaces to flow through the computational mesh $[25,26,39,46,47,74,79,88,94,98,118]$. MMALE methods generalize the classical approaches in which the mesh either follows the material motion (Lagrangian approach) or is fixed in space (Eulerian approach). Mesh elements cut 

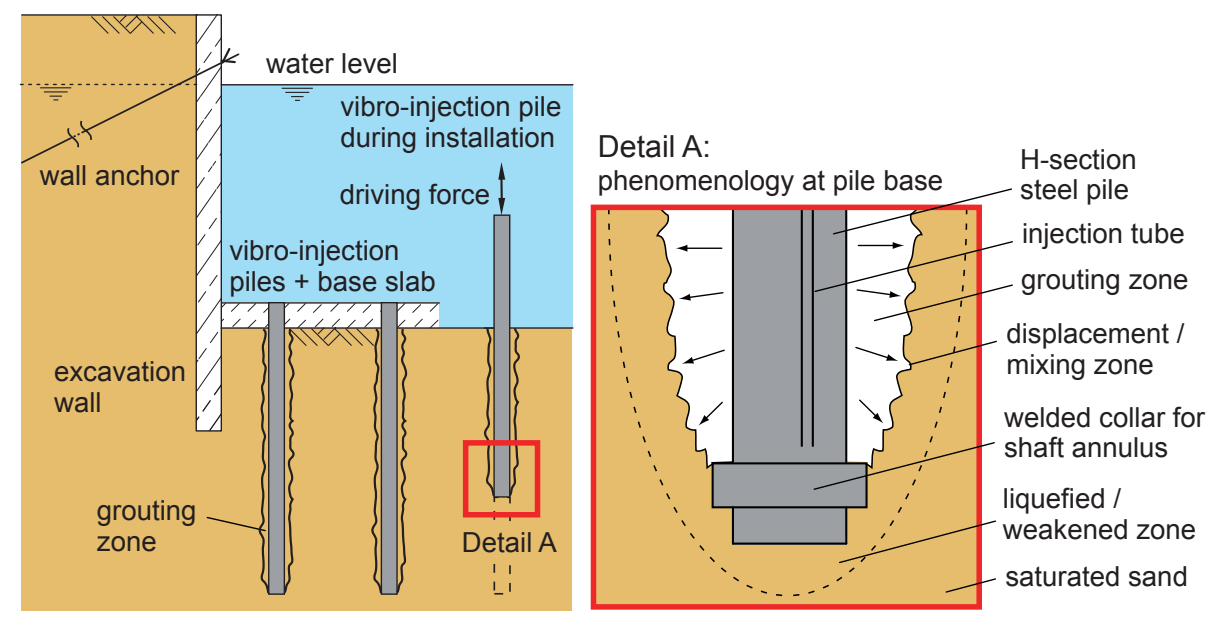

(a)

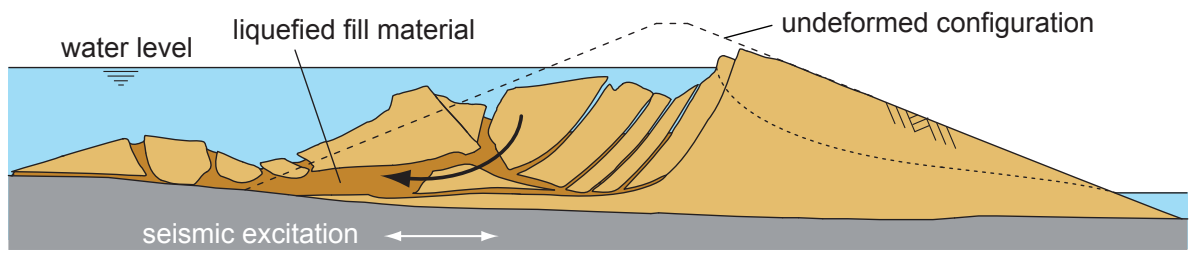

(b)

(c)

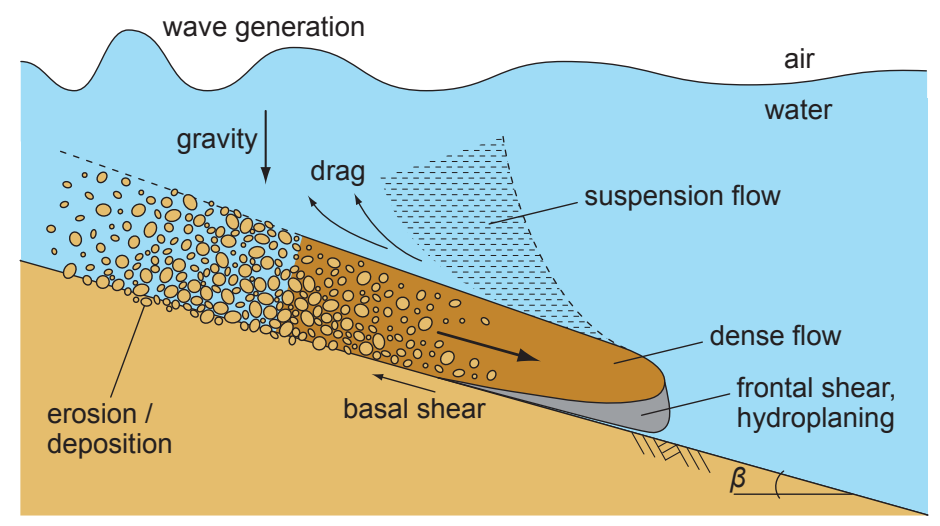

Fig. 1. Schematic of complex geomechanical multi-material flow situations. (a) Installation of vibro-injection piles to tie back the base slab of a deep excavation. (b) Liquefaction-induced failure of an earth-fill dam under seismic excitation; in accordance with [104]. (c) Submarine landslide; in accordance with [73]. 
by interfaces necessarily arise which contain a heterogeneous mixture of two or more materials. Because the mixture must be represented as an effective single-phase material (homogenized mixture), the underlying mixture theory is an essential ingredient of such methods for multi-material flow.

Besides the characteristics common to all multi-material flows, geomechanical multi-material flows are characterized by a complex coupled behavior of the saturated granular material representing the soil or debris material as well as by a hierarchy of distinct spatial scales (grain diameter, scale of mixture continuum representation, characteristic size of bulk material interfaces, etc.). While certain aspects of geomechanical multi-material flow can be considered as well understood, a fully-fledged flow model that is able to predict a time history of the material states for arbitrary compositions and configurations of the mixture is yet missing.

In two previous papers $[14,98]$ we have developed an MMALE finite element method accounting for the two-phase coupled response of saturated sand. The present paper goes into more detail about the three main features of the MMALE method for geomechanical problems, which are (i) the mixture theory for multi-material elements, (ii) the determination of the stress field, and (iii) the technique to resolve material interfaces. Accordingly, the paper has the following structure. Section 2 provides an overview of the MMALE method. The mathematical modeling of three-scale mixtures is addressed in Section 3, where we summarize the special mixture theory and the homogeneous equilibrium model derived in [14]. In Section 4 we focus on stress decompositions in saturated granular material resp. dense granular suspensions which enable the description of those arbitrary compositions and mixture configurations present in geomechanical multi-material flow. Section 5 is concerned with the treatment of material interfaces and their evolution (motion) from a non-Lagrangian point of view, i.e. in multi-material elements. After deriving the governing equations and introducing the basic discretization techniques, we outline the volume of fluid (VOF) interface reconstruction and propagation methods implemented into our MMALE code and present some preliminary results. The paper closes with concluding remarks and outlook in Section 6.

\section{Overview of the MMALE Method}

Our multi-material method is an extension of the single-material or simplified arbitrary Lagrangian-Eulerian (ALE) approach [9, 10, 12, 13,97]. A detailed description is given in [98], so only the basic equations will be presented in this section. The continuum mechanical background can be found in $[9,19,76,113,114]$.

The MMALE method addresses isothermal mechanical initial boundary value problems which are governed by conservation of mass

$$
\dot{\rho}+\rho \operatorname{div} \boldsymbol{v}=0,
$$

and balance of momentum

$$
\rho \dot{\boldsymbol{v}}=\rho \boldsymbol{b}+\operatorname{div} \boldsymbol{\sigma} .
$$


The equations are written in updated Lagrangian form referring to the spatial domain $\mathcal{D} \subset \mathbb{R}^{3}$ instantaneously occupied by the materials at time $t \in[0, T]$. They are assumed to hold at all points $x \in \mathcal{D}$ and for all $t \in[0, T]$. The field $\boldsymbol{v}=\boldsymbol{u}$ is the spatial image of the material velocity, $\boldsymbol{u}$ is the material displacement, $\rho$ is the spatial mass density, $\boldsymbol{b}$ is a prescribed body force per unit mass (e.g. gravitational acceleration), and $\boldsymbol{\sigma}=\boldsymbol{\sigma}^{\mathrm{T}}$ is the symmetric Cauchy stress. The superscribed $\mathrm{T}$ refers to the transpose. Moreover, the superposed dot is shorthand for the material time derivative $\dot{q}=\frac{\partial}{\partial t} q+\boldsymbol{v} \cdot \boldsymbol{\nabla} q$ of a time-dependent spatial field $q$, div is the spatial divergence operator, $\otimes$ is the tensor product, and $\cdot$ denotes the single contraction of tensors.

The stress tensor is decomposed into a pressure stress and an extra stress according to

$$
\boldsymbol{\sigma}=-p \boldsymbol{I}+\boldsymbol{s},
$$

where $p$ is the pressure and $\boldsymbol{I}$ is the second-order unit tensor. We assume that the extra stress is always deviatoric such that $p=-\frac{1}{3} \operatorname{tr} \boldsymbol{\sigma}$ and $\boldsymbol{s}=\boldsymbol{\sigma}_{\mathrm{dev}}$, where $\sigma_{\mathrm{dev}}=\boldsymbol{\sigma}-\frac{1}{3}(\operatorname{tr} \boldsymbol{\sigma}) \boldsymbol{I}$ is the deviatoric stress, $\operatorname{tr} \boldsymbol{a}=\boldsymbol{I}: \boldsymbol{a}$ returns the trace of a second-order tensor $\boldsymbol{a}$, and : indicates double contraction.

The rate of pressure is related to the rate of mass density through a compression model

$$
-\left.\frac{1}{V} \frac{\partial V}{\partial p}\right|_{M}=\frac{1}{\rho} \frac{\mathrm{d} \rho}{\mathrm{d} p}=\frac{1}{K} \quad \text { resp. } \quad \dot{p}=\frac{K}{\rho} \dot{\rho} .
$$

$K$ is the bulk modulus, $V$ and $M=\rho V$ are the volume and mass of a bounded region, respectively, and $\left.\right|_{M}$ means that mass is kept constant along with differentiation.

The balance equations (1) and (2) are rewritten using (3) and (4), treating $\boldsymbol{v}$ and $p$ as the independent variables:

$$
\begin{aligned}
\rho \dot{\boldsymbol{v}}-\rho \boldsymbol{b}-\operatorname{div}(\boldsymbol{s}-p \boldsymbol{I}) & =\mathbf{0}, \\
\dot{p}+K \operatorname{div} \boldsymbol{v} & =0 .
\end{aligned}
$$

The ALE formulation $[8-10,13,24,57]$ introduces a reference domain which may move in space at an arbitrary velocity $\boldsymbol{w}$. This velocity is referred to as the mesh velocity because the reference domain is represented by the computational mesh in numerical implementation. The difference $\boldsymbol{c}=\boldsymbol{v}-\boldsymbol{w}$ is called the convective velocity. The relative volume change between the referential coordinate system and the spatial coordinate system is the Jacobian, $J$, and its rate of change is given by

$$
\frac{\partial J}{\partial t}=J \operatorname{div} \boldsymbol{w} .
$$

Moreover, if $\hat{q}$ is the description of a spatial field $q$ in the referential coordinates, then the rate of $\hat{q}$ is related to the material time derivative through

$$
\dot{q}=\frac{\partial \hat{q}}{\partial t}+\boldsymbol{c} \cdot \nabla q .
$$


Substitution of (8) into the equations (5) and (6), respectively, and using the product rule yields

$$
\begin{aligned}
\frac{\partial \hat{\rho} \hat{\boldsymbol{v}}}{\partial t}+\operatorname{div}(\rho \boldsymbol{v} \otimes \boldsymbol{c})+\rho \boldsymbol{v} \operatorname{div} \boldsymbol{w}-\rho \boldsymbol{b}-\operatorname{div}(\boldsymbol{s}-p \boldsymbol{I}) & =\mathbf{0}, \\
\frac{\partial \hat{p}}{\partial t}+\operatorname{div}(p \boldsymbol{c})-p \operatorname{div} \boldsymbol{c}+K \operatorname{div} \boldsymbol{v} & =0 .
\end{aligned}
$$

Multiplication with $J$, substitution of (7), and arranging terms then results in the ALE conservation form of (5) and (6),

$$
\begin{aligned}
\frac{\partial \hat{\rho} \hat{\boldsymbol{v}} J}{\partial t}+J \operatorname{div}(\rho \boldsymbol{v} \otimes \boldsymbol{c}) & =J(\rho \boldsymbol{b}+\operatorname{div}(\boldsymbol{s}-p \boldsymbol{I})), \\
\frac{\partial \hat{p} J}{\partial t}+J \operatorname{div}(p \boldsymbol{c}) & =J(p-K) \operatorname{div} \boldsymbol{v} .
\end{aligned}
$$

We write this set of equations in the compact form

$$
\frac{\partial \hat{q} J}{\partial t}+J \operatorname{div} F=S J,
$$

where $q \in\{\rho \boldsymbol{v}, p\}, F$ is the convective flux of $q$, and $S$ is the source term.

The MMALE method is based on the common Lagrange-remap strategy which divides the incremental solution of the nonlinear problem into a Lagrangian step and remap step (Fig. 2). Conceptually, (13) is split into two sets of equations which are solved sequentially:

$$
\begin{aligned}
\frac{\partial \hat{q} J}{\partial t} & =S J, \\
\frac{\partial \hat{q} J}{\partial t}+J \operatorname{div} F & =0 .
\end{aligned}
$$

The first set of equations, (14), is associated with $\boldsymbol{c}=\mathbf{0}$ resp. $\boldsymbol{v}=\boldsymbol{w}$. Hence, it is equivalent to the set of equations (5) and (6) and formalizes a Lagrangian description of motion. During the Lagrangian step the set (14) is solved with standard finite element methods for the two-field mixed element formulation by accounting for large deformations [71,117,121]. Accordingly, (5) and (6) are written in a weak form which is discretized in space using finite elements. The solution of the semi-discrete weak form of the governing equations is advanced implicitly in time using the Newmark-beta and generalized trapezoidal methods in conjunction with a damped Newton-Raphson method.

The solution of the second set of equations, (15), is associated with the remap step. The remap step first relocates the nodes to reduce mesh distortion and then transfers the solution variables onto the modified mesh by using a conservative advection algorithm $[9,13]$. Time is advanced only during the Lagrangian step, whereas the spatial distributions of the solution variables are fixed during the remap step. That is,

$$
\boldsymbol{v} \equiv \mathbf{0} \quad \text { and } \quad \frac{\partial q}{\partial t} \equiv 0, \quad \text { but } \quad \boldsymbol{c} \neq \mathbf{0},
$$




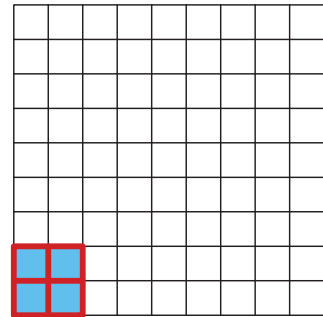

initial configuration

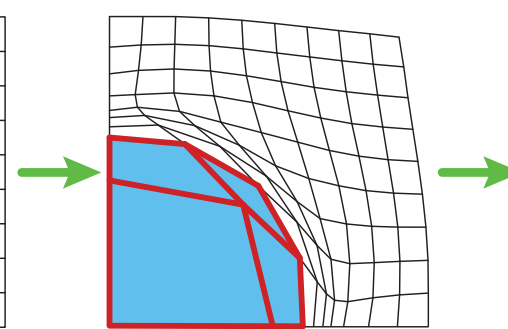

after the Lagrangian step

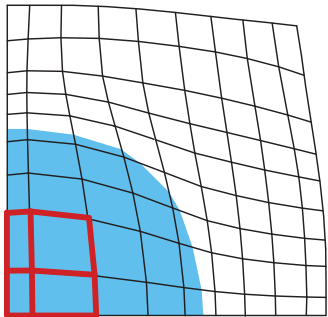

after the remap step

Fig. 2. Schematic diagram of the Lagrange-remap strategy in a calculational cycle of the MMALE method [98]. The blue area indicates a material zone whose initial configuration is assigned to an element patch highlighted in red. In the Lagrangian step the governing equations are solved with respect to the mesh deforming with the material. During the remap step the mesh distortion is reduced and the solution variables are transferred to the modified mesh. After the remap step several elements intersect with the material interface. These elements contain a mixture of two materials (blue and white) and are called multi-material elements.

so that $\dot{q}=0$ holds but $\boldsymbol{\nabla} \neq \neq \mathbf{0}$ in general. The overall Lagrange-remap solution procedure of the MMALE method is summarized in Alg. 1.

Because the reference domain (finite element mesh) is moved relative to the "frozen" material during the remap step, elements may arise in MMALE methods which intersect with material interfaces and thus contain a mixture of two or more materials (Fig. 2). However, the spatial distribution of the elements's degrees of freedom is homogeneous, so that a lack of information arises within these multi-material elements. The main difficulties are to accurately determine the states of the individual material portions and the reaction of the element they will generate [103]. This is particularly true for geomechanical multi-material flows which exhibit several spatial scales. For that reason, we have developed a three-scale mixture theory and derived a homogeneous equilibrium model which provides reasonable, physically-based mixing rules. These are summarized in the following section; the details are presented elsewhere $[11,14]$.

\section{Mathematical Modeling of Three-Scale Mixtures}

\subsection{Averaging Procedure}

The three-scale (micro, meso, and macro) system of interest is illustrated in Fig. 3. A still image of the flow recorded through a spatially fixed and reasonably small observation window is shown above in that figure. The flow consists of a bulk solid (S), a bulk fluid (F), and a composite material representing a fluidsaturated granular material $(\mathrm{G})$. The granular material by itself is an immiscible 


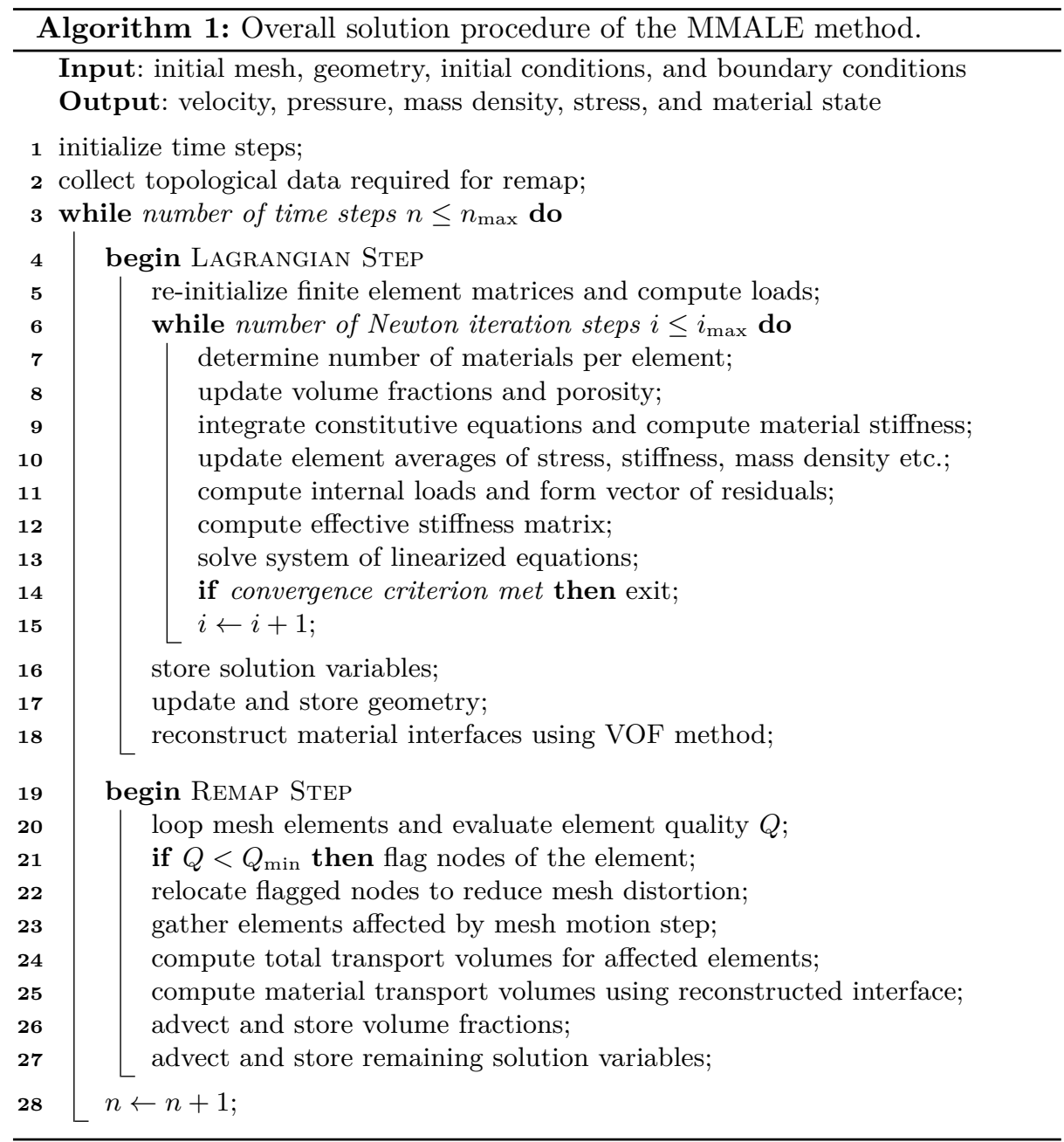




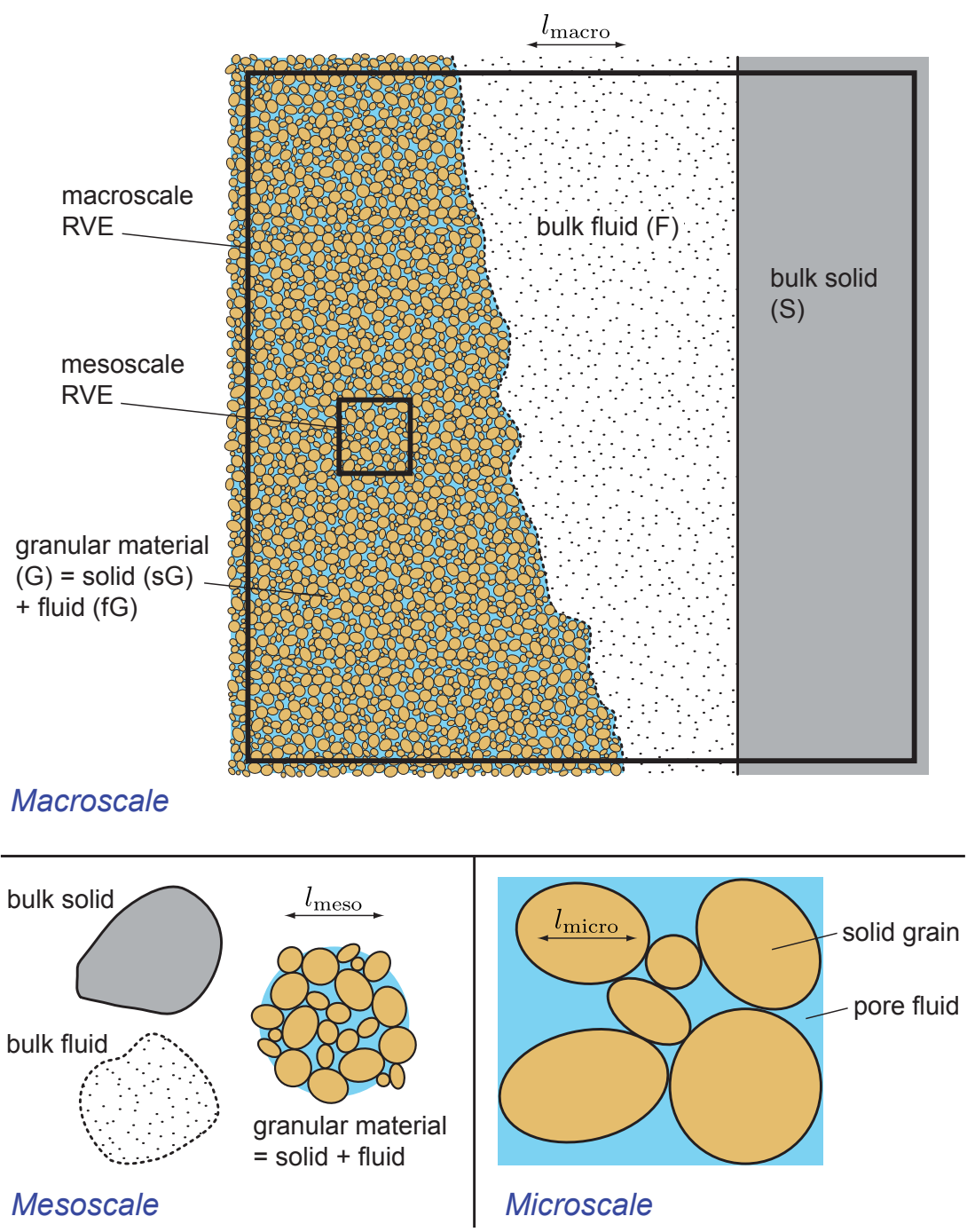

Fig. 3. Three spatial scales in a particular geomechanical multi-material flow. 
mixture consisting of a solid phase (s) and fluid phase (f). Void is considered as a particular fluid.

The granular material is constituted by an assembly of solid grains, whose typical diameter defines the microscale of the problem, $l_{\text {micro }}$ (Fig. 3 below). The characteristic length at which the grain assembly can be represented by a continuum is introduced as the mesoscale $l_{\text {meso }}$. At the mesoscale the bulk solid, the bulk fluid, and granular material can be regarded as homogeneous materials separated by sharp interfaces. Finally, at the macroscale the immiscible mixture of mesoscale continua (bulk solid, bulk fluid, and granular material) can be equivalently modeled as an effective single-phase material (homogenized immiscible mixture). Hence, we assume that the multi-material flow has a representative volume element (RVE) with characteristic length $l_{\text {macro }}$ (Fig. 3 above). The real world problem is modeled on an even larger scale.

To keep our theory as general as possible, each material $k \in\{\mathrm{S}, \mathrm{F}, \mathrm{G}\} \stackrel{\text { def }}{=}$ $\{1, \ldots, M\}$ is initially viewed as containing the same phases $\alpha \in\{\mathrm{s}, \mathrm{f}\} \stackrel{\text { def }}{=}$ $\{1, \ldots, N\}$, even though the fractional volume of one phase in a particular material might be zero. This means, for example, that the bulk solid is initially viewed as being composed of a solid phase and a fluid phase, even though the volume fraction of the fluid is zero. A particular phase $\alpha$ in a particular material $k$ represents an individual, chemically-independent constituent of the flow and will be denoted by $\alpha k$. For the particular flow under consideration we write $\mathrm{sS} \equiv \mathrm{S}$ and $\mathrm{fF} \equiv \mathrm{F}$ such that $\alpha k \in\{\mathrm{S}, \mathrm{F}, \mathrm{sG}, \mathrm{fG}\}$.

The flow takes place in a time interval $[0, T] \subset \mathbb{R}$ and in a three-dimensional modeling domain $\mathcal{D} \subset \mathbb{R}^{3}$ of the ambient Euclidian space. The subregions in $\mathcal{D}$ instantaneously occupied by the $k$-material and the $\alpha$-phase at time $t \in[0, T]$ are denoted by $\mathcal{M}^{k}$ and $\mathcal{P}^{\alpha}$, respectively, with

$$
\mathcal{D}=\bigcup_{\alpha} \mathcal{P}^{\alpha}=\bigcup_{k} \mathcal{M}^{k} .
$$

The (possibly empty) domain of the $\alpha$-phase in the $k$-material is given by the intersection $\mathcal{P}^{\alpha} \cap \mathcal{M}^{k}$. Based on the assumptions above, each two phases and each two materials do only intersect at their interface (if any).

Let $\chi^{k}$ be the material indicator function on $\mathcal{M}^{k} \subset \mathcal{D}$ and $\chi^{\alpha}$ the phase indicator function on $\mathcal{P}^{\alpha} \subset \mathcal{D}$, with $\chi^{k}, \chi^{\alpha}: \mathcal{D} \times[0, T] \rightarrow\{0,1\}$. The product of $\chi^{k}$ and $\chi^{\alpha}$ defines another indicator function which picks out the generally timedependent $\alpha$-phase domain of the $k$-material domain in the modeling domain:

$$
\chi^{\alpha k}(x, t) \stackrel{\text { def }}{=}\left(\chi^{\alpha} \chi^{k}\right)(x, t)= \begin{cases}1 & \text { if } x \in\left(\mathcal{P}^{\alpha} \cap \mathcal{M}^{k}\right) \text { at time } t, \\ 0 & \text { if } x \in \mathcal{D} \backslash\left(\mathcal{P}^{\alpha} \cap \mathcal{M}^{k}\right) \text { at time } t .\end{cases}
$$

This indicator function is unique to our mixture theory. It covers arbitrary flow compositions bounded between the classical cases of mixtures composed of singlephase materials $\left(\chi^{\alpha}=1\right)$ and mixtures represented by a single multiphase material $\left(\chi^{k}=1\right)$.

Indicator functions represent distributions, hence possess a weak derivative. Therefore, in accordance with equations (17)-(22) of [41], it can be shown that 
the substantial time derivative of $\chi^{\alpha k}$ defined by (18) is given by the topological equation

$$
\frac{\partial \chi^{\alpha k}}{\partial t}+\boldsymbol{v}_{\mathrm{I}}^{\alpha k} \cdot \nabla \chi^{\alpha k}=0, \quad \text { with } \quad \nabla \chi^{\alpha k}=\delta_{\mathrm{I}}^{\alpha k} \boldsymbol{n}_{\mathrm{I}}^{\alpha k}
$$

Here $\boldsymbol{v}_{\mathrm{I}}^{\alpha k}$ is the velocity of the $\alpha$-phase- $k$-material interface $\partial\left(\mathcal{P}^{\alpha} \cap \mathcal{M}^{k}\right), \boldsymbol{n}_{\mathrm{I}}^{\alpha k}$ is the field of outward normals on that interface, and $\delta_{\mathrm{I}}^{\alpha k}$ is a Dirac delta function which picks out the $\alpha$-phase- $k$-material interface in $\mathcal{D}$. Accordingly, $\nabla \chi^{\alpha k}$ is everywhere zero except for $\partial\left(\mathcal{P}^{\alpha} \cap \mathcal{M}^{k}\right)$.

Upscaling information from lower to higher scales can be achieved by different types of approaches, and each has its advantages and disadvantages $[18,37,55]$. The approach followed here is known as hybrid mixture theory $[1,21,22,54,55]$. The basic idea is to apply local volume averaging $[32,41,42]$ to the small scale balance equations and to make the constitutive assumptions needed for closure at the large scale, that is, for the averaged balance equations. The closure relations can be obtained either by direct postulation of desirable equations, as done in [71] and in this paper, or based on thermodynamical considerations as in the continuum theory of mixtures and the theory of porous media $[34,40,113]$.

Local local volume averaging is carried out with respect to a macroscopic RVE of the flow at all points $x \in \mathcal{D}$, which is defined through a spatially fixed and timeindependent subset $\mathcal{H}(x) \subset \mathbb{R}^{3}$. At each instant $t$, the RVE intersects with the current configuration of materials and phases as well as with their boundaries. The subregion of the RVE occupied by the $k$-material is $\mathcal{M}^{k} \cap \mathcal{H} \stackrel{\text { def }}{=} \mathcal{H}^{k}$, and $\left(\mathcal{P}^{\alpha} \cap \mathcal{M}^{k}\right) \cap \mathcal{H} \stackrel{\text { def }}{=} \mathcal{H}^{\alpha k}$ is the subregion occupied by the $\alpha$-phase of the $k$ material, with $\mathcal{H}=\bigcup_{k} \mathcal{H}^{k}=\bigcup_{k} \bigcup_{\alpha} \mathcal{H}^{\alpha k}$ by (17). The $\mathcal{H}$-average of an arbitrary time-dependent spatial microscopic field $q(x, t)$ is then defined through

$$
\langle q\rangle(x, t) \stackrel{\text { def }}{=} \frac{1}{H} \int_{\mathcal{H}} q(x+\boldsymbol{a}, t) \mathrm{d} v \quad \text { for all } \boldsymbol{x} \in \mathcal{D} \text { and } t \in[0, T],
$$

in which $\boldsymbol{a}$ is a vector on $\mathcal{H}, \mathrm{d} v$ is the volume density on $\mathbb{R}^{3}$, and $H \stackrel{\text { def }}{=} \int_{\mathcal{H}} 1 \mathrm{~d} v=$ const is the volume measure of $\mathcal{H}$.

Particular examples of the local volume average defined by (20) are the volume fractions

$$
f^{k} \stackrel{\text { def }}{=}\left\langle\chi^{k}\right\rangle=\frac{H^{k}}{H} \quad \text { and } \quad \pi^{\alpha k} \stackrel{\text { def }}{=} \frac{1}{f^{k}}\left\langle\chi^{\alpha k}\right\rangle=\frac{H^{\alpha k}}{H^{k}} \quad \in[0,1],
$$

where $H^{k} \stackrel{\text { def }}{=} \int_{\mathcal{H}^{k}} 1 \mathrm{~d} v=\int_{\mathcal{H}} \chi^{k} \mathrm{~d} v$ and $H^{\alpha k} \stackrel{\text { def }}{=} \int_{\mathcal{H}^{\alpha k}} 1 \mathrm{~d} v=\int_{\mathcal{H}} \chi^{\alpha k} \mathrm{~d} v$. While $f^{k}$ is the volume fraction of the $k$-material with respect to the RVE, $\pi^{\alpha k}$ represents the macroscale volume fraction of the $\alpha$-phase intrinsic to the $k$-material. The topology present in $\mathcal{H}$ entails the fundamental properties

$$
\sum_{k} f^{k}=1 \quad \text { and } \quad \sum_{\alpha} \pi^{\alpha k}=1 \quad \text { for all } k \in\{1, \ldots, M\}
$$


since phase or material overlaps are precluded. If the physical field $q(x, t)$ is defined per unit volume, then

$$
\langle q\rangle=\sum_{k} f^{k} q^{k}=\sum_{k} \sum_{\alpha} f^{k} \pi^{\alpha k} q^{\alpha k}, \quad \text { with } \quad q^{\alpha k} \stackrel{\text { def }}{=} \frac{\left\langle\chi^{\alpha k} q\right\rangle}{f^{k} \pi^{\alpha k}},
$$

follows immediately from (22) and the averaging operator (20). For example, if $q=\rho$ is the microscopic spatial mass density, then the intrinsic or true mass density $\rho^{\alpha k}$ represents the mass of the constituent $\alpha k$ per unit volume of that constituent, $\pi^{\alpha k} \rho^{\alpha k}$ is the mass of the constituent $\alpha k$ per unit volume of the $k$-material, and $f^{k} \pi^{\alpha k} \rho^{\alpha k}$ denotes its mass per unit volume of the mixture. Therefore, the latter two are bulk mass densities.

\subsection{Macroscopic Balance Equations}

On the microscale all constituents of the mixture are regarded as compressible continua, governed by the equations of continuum mechanics $[76,113,114]$. The balance principles of the problems under consideration are conservation of mass, (1), and balance of momentum, (2), in conjunction with the interface jump conditions. We currently do not take care of any thermodynamical issue. Mass is neither supplied in the interior of any constituent nor at the interfaces. Moreover, there is no interfacial momentum supply due to surface tension.

Each term of the microscopic balance equations is averaged by using the procedure outlined in the previous section; see [11, 14, 41, 42] for details. This results in the $\alpha$-phase- $k$-material macroscopic conservation of mass

$$
\frac{\partial f^{k} \pi^{\alpha k} \rho^{\alpha k}}{\partial t}+\operatorname{div}\left(f^{k} \pi^{\alpha k} \rho^{\alpha k} \boldsymbol{v}^{\alpha k}\right)=\Lambda^{\alpha k}
$$

and macroscopic balance of momentum

$$
\begin{array}{r}
\frac{\partial f^{k} \pi^{\alpha k} \rho^{\alpha k} \boldsymbol{v}^{\alpha k}}{\partial t}+\operatorname{div}\left(f^{k} \pi^{\alpha k} \rho^{\alpha k} \boldsymbol{v}^{\alpha k} \otimes \boldsymbol{v}^{\alpha k}\right)= \\
f^{k} \pi^{\alpha k} \rho^{\alpha k} \boldsymbol{b}^{\alpha k}+\operatorname{div}\left(f^{k} \pi^{\alpha k} \boldsymbol{\sigma}^{\alpha k}\right)+\Lambda^{\alpha k} \boldsymbol{v}_{\mathrm{I}}^{\mathrm{m}}+\boldsymbol{\Gamma}^{\alpha k}
\end{array}
$$

where

$$
\begin{aligned}
& \Lambda^{\alpha k} \stackrel{\text { def }}{=}\left\langle\left(\rho\left(\boldsymbol{v}-\boldsymbol{v}_{\mathrm{I}}\right)\right)^{[\alpha k]} \cdot \boldsymbol{n}_{\mathrm{I}}^{\alpha k}\right\rangle, \\
& \Lambda^{\alpha k} \boldsymbol{v}_{\mathrm{I}}^{\mathrm{m}} \stackrel{\text { def }}{=}\left\langle\left(\rho \boldsymbol{v} \otimes\left(\boldsymbol{v}-\boldsymbol{v}_{\mathrm{I}}\right)\right)^{[\alpha k]} \cdot \boldsymbol{n}_{\mathrm{I}}^{\alpha k}\right\rangle, \quad \text { and } \\
& \boldsymbol{\Gamma}^{\alpha k} \stackrel{\text { def }}{=}-\left\langle\boldsymbol{\sigma}^{[\alpha k]} \cdot \boldsymbol{n}_{\mathrm{I}}^{\alpha k}\right\rangle \text {. }
\end{aligned}
$$

The superscribed $\alpha k$ denotes macroscopic (i.e. $\mathcal{H}$-averaged) fields related to the $\alpha$-phase in the $k$-material. The mass transfer term $\Lambda^{\alpha k}$ denotes the rate of mass supply per unit volume via the $\alpha$-phase- $k$-material interface. The momentum transfer term $\boldsymbol{\Gamma}^{\alpha k}$ includes drag forces per unit volume generated by the relative motion of the constituents. Note that $\Gamma^{\alpha k}$ accounts for surface forces, 
but not for momentum exchange owing to transfer of inertial mass which is described by the term $\Lambda^{\alpha k} \boldsymbol{v}_{\mathrm{I}}^{\mathrm{m}}$. Total mass and momentum of the mixture is conserved. Hence, the sum of the transfer terms over all constituents must vanish:

$$
\sum_{k} \sum_{\alpha} \Lambda^{\alpha k}=0 \quad \text { and } \quad \sum_{k} \sum_{\alpha}\left(\Lambda^{\alpha k} \boldsymbol{v}_{\mathrm{I}}^{\alpha k}+\boldsymbol{\Gamma}^{\alpha k}\right)=\mathbf{0} .
$$

From this and the conditions (23), summation of (24) and (25) over all phases $\alpha \in\{1, \ldots, N\}$ and all materials $k \in\{1, \ldots, M\}$ finally yield the macroscopic conservation of mass and macroscopic balance of momentum of the mixture:

$$
\frac{\partial\langle\rho\rangle}{\partial t}+\operatorname{div}\langle\rho \boldsymbol{v}\rangle=0 \quad \text { and } \quad \frac{\partial\langle\rho \boldsymbol{v}\rangle}{\partial t}+\operatorname{div}\langle\rho \boldsymbol{v} \otimes \boldsymbol{v}\rangle=\langle\rho \boldsymbol{b}\rangle+\operatorname{div}\langle\boldsymbol{\sigma}\rangle .
$$

The macroscopic balance equations (30) in conjunction with the jump conditions (29) and the balance equations (24) and (25) provide unified description of non-reactive isothermal flow of an immiscible mixture of $M$ materials consisting of $N$ phases. They hold at each spatial point and at all interfaces and refer to a spatial reference volume instantaneously occupied by the mixture on the macroscale. A single spatial point is viewed as being simultaneously occupied by all materials and all phases, that is, the mixture after averaging is viewed as being composed of overlapping continua.

The equations explicitly account for volume fractions of each bulk material and for volume fractions of each phase in the bulk materials. Moreover, the equations include separate physical quantities for each constituent and separate terms representing the interaction between the constituents. The mechanical behavior of the mixture is a consequence of the mechanical behavior of its individual constituents, their volume fractions as well as of their interactions. Therefore, the macroscopic equations can explicitly represent diverse compositions or evolving configurations of multi-material flow.

\subsection{Homogeneous Equilibrium Model}

The particular geomechanical multi-material flow of interest can be locally described as a mixture consisting of a bulk solid (S), a bulk fluid (F), and a fluid-saturated granular material $(\mathrm{G})$ composed of a solid phase (sG) and a fluid phase (fG); cf. Fig. 3. Hence, the materials generally represent binary immiscible mixtures, solely composed of a solid phase and a fluid phase such that $\alpha \in\{\mathrm{s}, \mathrm{f}\}$. We denote the fluid fraction or porosity of the $k$-material, $k \in\{\mathrm{S}, \mathrm{F}, \mathrm{G}\}$, by

$$
n^{k} \stackrel{\text { def }}{=} \pi^{\mathrm{f} k},
$$

so the solid fraction within the $k$-material becomes $\pi^{\mathrm{s} k}=1-n^{k}$ by using $(22)_{2}$. We remark that in cases where the $k$-material consists of a solid without significant porosity $(k=\mathrm{S})$ one has $n^{\mathrm{S}}=0$. If on the other hand the $k$-material is a fluid $(k=\mathrm{F})$, then $n^{\mathrm{F}}=1$ applies. The mixture represented by a single fluidsaturated granular material is characterized by $f^{k} \equiv f^{\mathrm{G}}=1$ and $0<n^{\mathrm{G}}<1$.

Modeling of a particular multi-material flow requires closure of the set of balance equations, which is otherwise underdetermined. Generally the following closure relations have to be specified $[32,33]$ : 
1. Transfer relations expressing the physics at the material interfaces.

2. Topological relations accounting for the evolution of the interfacial structure.

3. Constitutive relations characterizing the physical behavior of each material.

Concerning the first group of closure relations we take the simplest approach by assuming zero mass and momentum exchange, so that $\Lambda^{\alpha k}=0$ and $\boldsymbol{\Gamma}^{\alpha k}=\mathbf{0}$ for all $\alpha \in\{\mathrm{s}, \mathrm{f}\}$ and $k \in\{\mathrm{S}, \mathrm{F}, \mathrm{G}\}$. The former complies with the assumption of no phase change and no chemical reaction at interfaces. The assumption of zero momentum transfer, on the other hand, may contradict flow situations in reality in which interactions, e.g. based on viscous drag, play an important role. Moreover, a granular material in the mixture, by this assumption, must be either dry $\left(\rho^{\mathrm{fG}}=0\right)$ or locally undrained (no consolidation effects).

The topological closure relations restore the information of the flow structure lost by the application of volume averaging $[32,33]$. For the flow situation under consideration, the only relations required are those that account for the evolution of the material volume fractions $f^{k}$. A proper closure relation for volume fraction has to specify how the volumetric distribution of the bulk solid, the bulk fluid, and the saturated granular medium evolves during the particular geomechanical multi-material flow under consideration. Because further research is needed to establish such a physics-based topological closure law, we have simply assumed homogeneous distributions of pressure and velocity between the materials:

$$
p^{k}=\langle p\rangle \quad \text { and } \quad \boldsymbol{v}^{k}=\langle\boldsymbol{v}\rangle \quad \text { for all } k \in\{\mathrm{S}, \mathrm{F}, \mathrm{G}\} \text { and } t \in[0, T] .
$$

From a physical viewpoint this means that everything is in homogeneous thermodynamic equilibrium [35,80], and its limitations are discussed in [14].

The description of material behavior and the development of constitutive relations are major concerns in continuum mechanics. Restrictions on the form of the closure relations result from the principles of constitutive theory $[42,76,114]$. In order to treat all materials and material compositions that might be present in geomechanical multi-material flow in a unified fashion, the stress tensor of any material is decomposed into a pressure stress $-p^{\alpha k} \boldsymbol{I}$ and an extra stress $\boldsymbol{s}^{\alpha k}[76,114]$, in accordance with $(3)$ :

$$
\boldsymbol{\sigma}^{\alpha k}=-p^{\alpha k} \boldsymbol{I}+\boldsymbol{s}^{\alpha k}
$$

We assume that the extra stress is always deviatoric such that $p^{\alpha k}=-\frac{1}{3} \operatorname{tr} \boldsymbol{\sigma}^{\alpha k}$, and that all constituents of the flow are compressible, including both the grains and the fluid phase of the granular material.

A rather long but almost straightforward derivation using all the ingredients yields the following homogeneous equilibrium model for geomechanical multimaterial flow which is consistent with the set of equations (5) and (6) for a single-material problem $[11,14]$ :

$$
\begin{aligned}
\langle\rho\rangle\langle\dot{\boldsymbol{v}}\rangle-\langle\rho \boldsymbol{b}\rangle-\operatorname{div}\langle\boldsymbol{s}-p \boldsymbol{I}\rangle & =\mathbf{0} \\
\langle\dot{p}\rangle+\langle K\rangle \operatorname{div}\langle\boldsymbol{v}\rangle & =0,
\end{aligned}
$$


where

$$
\begin{array}{r}
\langle\boldsymbol{s}\rangle=\sum_{k} f^{k} \boldsymbol{s}^{k}=f^{\mathrm{S}} \boldsymbol{s}^{\mathrm{S}}+f^{\mathrm{F}} \boldsymbol{s}^{\mathrm{F}}+f^{\mathrm{G}}\left(\boldsymbol{s}^{\mathrm{G}^{\prime}}+n^{\mathrm{G}} \boldsymbol{s}^{\mathrm{fG}}\right), \\
\langle p\rangle=\sum_{k} f^{k} p^{k}=f^{\mathrm{S}} p^{\mathrm{S}}+f^{\mathrm{F}} p^{\mathrm{F}}+f^{\mathrm{G}}\left(p^{\mathrm{G}^{\prime}}+p^{\mathrm{fG}}\right), \\
\langle\rho\rangle=\sum_{k} f^{k} \rho^{k}=f^{\mathrm{S}} \rho^{\mathrm{S}}+f^{\mathrm{F}} \rho^{\mathrm{F}}+f^{\mathrm{G}}\left(\left(1-n^{\mathrm{G}}\right) \rho^{\mathrm{sG}}+n^{\mathrm{G}} \rho^{\mathrm{fG}}\right), \\
\left.\frac{1}{\langle K\rangle}=\sum_{k} \frac{f^{k}}{K^{k}}=\frac{f^{\mathrm{S}}}{K^{\mathrm{S}}}+\frac{f^{\mathrm{F}}}{K^{\mathrm{F}}}+\frac{f^{\mathrm{G}}}{K^{\mathrm{G}}}, \quad \zeta^{\mathrm{G}}=1-\frac{K_{\mathrm{d}}^{\mathrm{G}}}{K^{\mathrm{sG}}}\right) \\
\text { and } K^{\mathrm{G}}=K_{\mathrm{dr}}^{\mathrm{G}}\left(1+\frac{\left(\zeta^{\mathrm{G}}\right)^{2}}{\zeta^{\mathrm{G}} K_{\mathrm{dr}}^{\mathrm{G}} / K^{\mathrm{sG}}+n^{\mathrm{G}}\left(K_{\mathrm{dr}}^{\mathrm{G}} / K^{\mathrm{fG}}-K_{\mathrm{dr}}^{\mathrm{G}} / K^{\mathrm{sG}}\right)}\right) .
\end{array}
$$

The model is closed by the constitutive equations for the bulk solid ( $\alpha k \equiv k=\mathrm{S})$, the bulk fluid ( $\alpha k \equiv k=\mathrm{F}$ ), the granular material $(k=\mathrm{G})$ including the solid phase $(\alpha k=\mathrm{sG})$ and fluid phase $(\alpha k=\mathrm{fG})$, by the evolution equations for the porosity,

$$
\dot{n}^{\mathrm{G}}=\left(1-n^{\mathrm{G}}\right)\left(\frac{\dot{p}^{\mathrm{sG}}}{K^{\mathrm{sG}}}+\operatorname{div}\langle\boldsymbol{v}\rangle\right), \quad \text { with } \quad \dot{p}^{\mathrm{sG}}=\dot{p}^{\mathrm{G}^{\prime}} \frac{K^{\mathrm{sG}}}{K_{\mathrm{dr}}^{\mathrm{G}}}+\dot{p}^{\mathrm{fG}},
$$

and volume fractions,

$$
\dot{f}^{k}=f^{k}\left(\frac{\langle K\rangle}{K^{k}}-1\right) \operatorname{div}\langle\boldsymbol{v}\rangle,
$$

and by the compression models for each constituent,

$$
\dot{\rho}^{\mathrm{S}}=\frac{\rho^{\mathrm{S}}}{K^{\mathrm{S}}}\langle\dot{p}\rangle, \quad \dot{\rho}^{\mathrm{F}}=\frac{\rho^{\mathrm{F}}}{K^{\mathrm{F}}}\langle\dot{p}\rangle, \quad \dot{\rho}^{\mathrm{SG}}=\frac{\rho^{\mathrm{SG}}}{K^{\mathrm{sG}}} \dot{p}^{\mathrm{sG}}, \quad \dot{\rho}^{\mathrm{fG}}=\frac{\rho^{\mathrm{fG}}}{K^{\mathrm{fG}}} \dot{p}^{\mathrm{fG}} .
$$

Besides the quantities already defined, $p^{\mathrm{G}^{\prime}}$ and $s^{\mathrm{G}^{\prime}}$ are the effective pressure and effective deviatoric stress, respectively, in the granular material which will be defined in the subsequent section, $\langle K\rangle$ is the bulk modulus of the mixture, $K^{k}$ is the bulk modulus of the $k$-material, with $k \in\{\mathrm{S}, \mathrm{F}, \mathrm{G}\}, K^{\mathrm{sG}}$ and $K^{\mathrm{sG}}$ are intrinsic bulk moduli of the granular material solid phase and fluid phase, respectively, $K_{\mathrm{dr}}^{\mathrm{G}}$ is the bulk modulus of the drained granular material, $K_{\mathrm{uj}}^{\mathrm{G}} \approx$ $K^{\mathrm{sG}}$ is the unjacketed bulk modulus [20], and $\zeta^{\mathrm{G}}$ is the Biot-Willis coefficient $[29,30]$.

For the stress tensors in the bulk solid and in the bulk fluid the common constitutive equations can be substituted [19,42,76,114]; effects of turbulence in the bulk fluid are currently neglected. However, the stress tensor in the granular material $(k=\mathrm{G})$ needs to be analyzed in more detail. In particular, we have to justify our motivation to express the extra stress of the granular material in (36) by $s^{\mathrm{G}}=\boldsymbol{s}^{\mathrm{G}^{\prime}}+n^{\mathrm{G}} \boldsymbol{s}^{\mathrm{fG}}$. 


\section{Stress in Granular Materials and Suspensions}

The specific granular material of interest is a cohesionless granular material in which a single fluid fills the intersticial space. From a formal rheological viewpoint the material can be addressed as a dense (high concentration) grain-fluid mixture or "granular suspension" [3]. Our current research is particularly concerned with cohesionless soil (sand). The smallest diameter of the solid grains is generally larger than $0.075 \mathrm{~mm}$ and the solid volume fraction resp. volume concentration is basically higher than $50 \%$ (porosity $n^{\mathrm{G}}<0.5$ ) [38]. We assume for simplicity that the grains are permanent, i.e. they are non-abrasive and cannot crush. The fluid can be gas, liquid, or a suspension (slurry) of liquid and dispersed fines (grain diameter $<0.075 \mathrm{~mm}$ ).

According to $[7,59,96]$, two limiting regimes of dry granular flow have to be considered. Under static or quasi-static loads the grains are in close contact and form a network. The contact forces acting between the grains are dominated by the mean stress and dry friction (granular solid). Grain inertia effects are negligible, and the material response is rate-independent plastic. This is called the frictional or quasi-static regime, and it is the granular flow regime extensively studied in soil mechanics [102]. At the other extreme characterized by high rates of shear deformation and smaller solid volume fractions, the material behaves rate-dependent "viscous" (granular liquid). Grain inertia and instantaneous grain contacts through collision dominate [15], hence this flow regime is called the collisional or dynamic regime. In many practical flow situations frictional and collisional interactions are roughly of the same order, and the contributions of each to the bulk stress of the mixture cannot be clearly distinguished. However, relatively little is known about this intermediate flow regime, called the frictional-collisional regime, from both theoretical and experimental viewpoints $[4-6,65]$.

Further complexity is introduced by the interstitial fluid in granular materials. Fluid-solid coupling by Stokes' drag resulting from the relative velocity has been recognized for a long time in soil mechanics because it is responsible for consolidation $[29,110]$. Besides this, indirect grain interactions generally occur through lubricated contacts $[3,4,6,36]$. Lubricated contact is characterized by repulsive viscous forces due to squeezing and shearing of the interstitial fluid; the shear-thickening effect is an exemplary consequence of this phenomenon [36]. The rate of shear at which lubricational (or macros-viscous [15]) flow may take place at otherwise equal conditions ranges between those present in the frictional and collisional regimes. In general, all three flow regimes have to be considered in the analysis of debris flows [60,63] and liquefaction-induced flow of soils [69]. However, the description of the mechanical behavior of a dense grain-fluid mixture for a wide range of flow conditions and material properties is still an open problem [6].

Based on (23), the bulk stress in a saturated granular material can be generally expressed as

$$
\boldsymbol{\sigma}^{\mathrm{G}}=\left(1-n^{\mathrm{G}}\right) \boldsymbol{\sigma}^{\mathrm{sG}}+n^{\mathrm{G}} \boldsymbol{\sigma}^{\mathrm{fG}} \stackrel{\text { def }}{=} \tilde{\boldsymbol{\sigma}}^{\mathrm{sG}}+\tilde{\boldsymbol{\sigma}}^{\mathrm{fG}},
$$


with a fluid fraction resp. porosity $0<n^{\mathrm{G}}<1$, and taking $f^{\mathrm{G}} \equiv 1$. The tensors $\tilde{\boldsymbol{\sigma}}^{\mathrm{sG}}, \tilde{\boldsymbol{\sigma}}^{\mathrm{fG}}$ on the right are referred to as the partial stresses. These amalgamated stress tensors are used in many models for saturated porous media $[34,40,43]$ and debris flows $[6,60,63,64,90,91]$, particularly those derived from the continuum theory of mixtures.

In order to model the full frictional-collisional regime, the stress tensors of the solid and fluid phases are represented as the linear sum of a rate-independent frictional contribution and a rate-dependent viscous contribution $[7,59,60,116]$ :

$$
\boldsymbol{\sigma}^{\alpha \mathrm{G}} \stackrel{\text { def }}{=} \boldsymbol{\sigma}_{\mathrm{fr}}^{\alpha \mathrm{G}}+\boldsymbol{\sigma}_{\mathrm{vi}}^{\alpha \mathrm{G}}, \quad \text { with } \quad \alpha \in\{\mathrm{s}, \mathrm{f}\},
$$

so that $\boldsymbol{\sigma}^{\mathrm{G}}=\boldsymbol{\sigma}_{\mathrm{fr}}^{\mathrm{G}}+\boldsymbol{\sigma}_{\mathrm{vi}}^{\mathrm{G}}$ likewise. We then assume

$$
\operatorname{tr} \boldsymbol{\sigma}_{\mathrm{fr}}^{\mathrm{sG}}=-3 p^{\mathrm{sG}}, \quad \boldsymbol{\sigma}_{\mathrm{fr}}^{\mathrm{fG}}=-p^{\mathrm{fG}} \boldsymbol{I}, \quad \text { and } \quad \operatorname{tr} \boldsymbol{\sigma}_{\mathrm{vi}}^{\mathrm{fG}}=0 .
$$

The first assumption formalizes that the solid phase constituent is not subject to internal constraints [114]. The second is the interpretation of a saturated intersticial space [60], and the third assumption is because volume viscosity is usually neglected in porous media and debris flow theories. The latter two assumptions result in $\boldsymbol{\sigma}_{\mathrm{vi}}^{\mathrm{fG}}=\boldsymbol{s}^{\mathrm{fG}}$.

In accordance with [23], we introduce Terzaghi's effective stress $\boldsymbol{\sigma}_{\mathrm{fr}}^{\mathrm{G}^{\prime}}$ as the frictional partial stress of the solid phase in which the pressure has been replaced with the excess pressure $p^{\mathrm{sG}}-p^{\mathrm{fG}}$. Clearly,

$$
\frac{\boldsymbol{\sigma}_{\mathrm{fr}}^{\mathrm{G}^{\prime}}}{1-n^{\mathrm{G}}} \stackrel{\text { def }}{=}-\left(p^{\mathrm{sG}}-p^{\mathrm{fG}}\right) \boldsymbol{I}+\boldsymbol{s}_{\mathrm{fr}}^{\mathrm{sG}} \quad \text { and } \quad \frac{p^{\mathrm{G}^{\prime}}}{1-n^{\mathrm{G}}}=p^{\mathrm{sG}}-p^{\mathrm{fG}},
$$

where $p^{\mathrm{G}^{\prime}} \stackrel{\text { def }}{=}-\frac{1}{3} \operatorname{tr} \boldsymbol{\sigma}_{\mathrm{fr}}^{\mathrm{G}^{\prime}}$ is called the mean effective stress and $\boldsymbol{s}_{\mathrm{fr}}^{\mathrm{sG}}=\left(\boldsymbol{\sigma}_{\mathrm{fr}}^{\mathrm{sG}}\right)_{\mathrm{dev}}$ by $(46)_{1}$. Note that in a suspension without grain contacts each grain would be completely surrounded by water, resulting in $p^{\mathrm{sG}}=p^{\mathrm{fG}}$ and $p^{\mathrm{G}^{\prime}}=0$. In the light of (46) and (47) the total frictional (quasi-static) stress part of the saturated granular medium can be calculated from

$$
\boldsymbol{\sigma}_{\mathrm{fr}}^{\mathrm{G}}=\boldsymbol{\sigma}_{\mathrm{fr}}^{\mathrm{G}^{\prime}}-p^{\mathrm{fG}} \boldsymbol{I},
$$

which is known as Terzaghi's principle of effective stress [38, 110,121].

In contrast to the frictional part, we postulate that the effective stress for the collisional (dynamic) regime remains unaffected by fluid stresses, i.e. $\sigma_{\mathrm{vi}}^{\mathrm{G}^{\prime}}=$ $\left(1-n^{\mathrm{G}}\right) \boldsymbol{\sigma}_{\mathrm{vi}}^{\mathrm{sG}}$. Therefore, by taking into account (45), the assumptions (46), and the principle (48), the representation (44) of the total Cauchy stress can be recast into

$$
\begin{aligned}
\boldsymbol{\sigma}^{\mathrm{G}} & =\boldsymbol{\sigma}_{\mathrm{fr}}^{\mathrm{G}^{\prime}}+\boldsymbol{\sigma}_{\mathrm{vi}}^{\mathrm{G}^{\prime}}+\boldsymbol{\sigma}_{\mathrm{fr}}^{\mathrm{fG}}+n^{\mathrm{G}} \boldsymbol{\sigma}_{\mathrm{vi}}^{\mathrm{fG}} \\
& =\boldsymbol{\sigma}^{\mathrm{G}^{\prime}}-p^{\mathrm{fG}} \boldsymbol{I}+n^{\mathrm{G}} \boldsymbol{s}^{\mathrm{fG}},
\end{aligned}
$$

with $\boldsymbol{\sigma}^{\mathrm{G}^{\prime}}=\boldsymbol{\sigma}_{\mathrm{fr}}^{\mathrm{G}^{\prime}}+\boldsymbol{\sigma}_{\mathrm{vi}}^{\mathrm{G}^{\prime}}$. We refer to (49) as the principle of effective stress for a general saturated grain-fluid mixture. The same relation is used in [64] in a 
continuum mixture theory to describe the flow of variably fluidized granular masses (debris flow, rock avalanches, etc.).

Based on the general principle of effective stress (49), and the discussion at the beginning of this section concerned with the different flow regimes of dense grain-fluid mixtures, constitutive equations have to be specified for the fluid phase stress and for the frictional and viscous parts of the effective stress.

For simplicity, the interstitial fluid (pore fluid) is represented by a Newtonian fluid with deviatoric viscous stress. Hence, the constitutive behavior can be described by the standard Navier-Poisson relation together with the Stokes condition [76], leading to

$$
\boldsymbol{\sigma}^{\mathrm{fG}}=\boldsymbol{\sigma}_{\mathrm{fr}}^{\mathrm{fG}}+\boldsymbol{\sigma}_{\mathrm{vi}}^{\mathrm{fG}} \stackrel{\text { def }}{=}-p^{\mathrm{fG}} \boldsymbol{I}+2 \mu^{\mathrm{fG}} \boldsymbol{d}_{\mathrm{dev}}^{\mathrm{fG}}
$$

where $\boldsymbol{d} \stackrel{\text { def }}{=} \frac{1}{2}\left(\boldsymbol{\nabla} \boldsymbol{v}+(\boldsymbol{\nabla} \boldsymbol{v})^{\mathrm{T}}\right)$ is the spatial rate of deformation tensor and $\mu^{\mathrm{fG}}$ is the dynamic shear viscosity. Effects of turbulence are again neglected. In cases where the fluid phase does not represent pure liquid but a suspension with moderate concentrations of dispersed fines, estimates for $\mu^{\mathrm{fG}}$ can be found in [82]. Moreover, in order to account for the fact that the fluid phase fills the interstitial space of the distributed granular material we define $\mu^{\mathrm{fG}}\left(n^{\mathrm{G}}\right) \stackrel{\text { def }}{=}\left(n^{\mathrm{G}}\right)^{2} \mu_{0}^{\mathrm{fG}}$ in accordance with [86], where $\mu_{0}^{\mathrm{fG}}$ is the shear viscosity of the fluid for $n^{\mathrm{G}}=1$ (pure fluid).

Compared to the fluid phase, the mechanical behavior of cohesionless granular material is very complex and has several distinctive features $[48,59,60,68,99$, $102,111,121]$. Different approaches can be employed to model this behavior on the mesoscale. Here we are interested in exploring fundamental behavior of complex phenomena in geomechanics and geotechnical engineering. In this regard, phenomenological two-phase models relying on a continuum representation of granular material and not on micromechanics are eminently suited $[6,45,48,60,121]$. The application to general geomechanical multi-material flows calls for constitutive relations which need only a single set of material constants and then are able to simulate the mechanical behavior of granular material under complex loading paths over a wide range of densities and stress states. However, a constitutive relation accounting for all features and over the entire frictional-collisional regime is still out of reach.

Constitutive equations should be prescribed for the effective stress $\sigma^{\mathrm{G}^{\prime}}=$ $\boldsymbol{\sigma}_{\mathrm{fr}}^{\mathrm{G}^{\prime}}+\boldsymbol{\sigma}_{\mathrm{vi}}^{\mathrm{G}^{\prime}}$. Concerning the quasi-static frictional stress contribution $\boldsymbol{\sigma}_{\mathrm{fr}}^{\mathrm{G}^{\prime}}$, attractive models have been proposed for applications in soil mechanics and fall into the categories of elasto-plastic $[72,77,87,109]$ or hypoplastic $[17,51,83,115]$ rate constitutive equations. All of them determine an objective rate of the effective stress as a function of the rate of deformation, the effective stress, the porosity $n^{\mathrm{G}}$ or void ratio $e^{\mathrm{G}} \stackrel{\text { def }}{=} n^{\mathrm{G}} /\left(1-n^{\mathrm{G}}\right)$, and a (possibly empty) set of additional state variables $\boldsymbol{h}^{\mathrm{G}} \stackrel{\text { def }}{=}\left\{h_{1}^{\mathrm{G}}, \ldots, h_{m}^{\mathrm{G}}\right\}$. As an example, we consider the 
generic rate constitutive equation

$$
\begin{aligned}
\stackrel{\boldsymbol{\sigma}}{\mathrm{fr}}^{\mathrm{G}^{\prime}} & \stackrel{\text { def }}{=} \boldsymbol{c}_{\mathrm{fr}}^{\mathrm{G}^{\prime}}\left(\boldsymbol{\sigma}_{\mathrm{fr}}^{\mathrm{G}^{\prime}}, n^{\mathrm{G}}, \boldsymbol{h}^{\mathrm{G}}\right):\left(\boldsymbol{d}^{\mathrm{sG}}-\dot{\varepsilon}^{\mathrm{sG}} \boldsymbol{I}\right) \\
& \stackrel{\text { def }}{=} \boldsymbol{\sigma}_{\mathrm{fr}}^{\mathrm{G}^{\prime \prime}}-\dot{\varepsilon}^{\mathrm{sG}} \boldsymbol{c}_{\mathrm{fr}}^{\mathrm{G}^{\prime}}: \boldsymbol{I},
\end{aligned}
$$

in which $\stackrel{\nabla}{\stackrel{\nabla}{\text { def }}=} \dot{\boldsymbol{\sigma}}+\boldsymbol{\sigma} \cdot \boldsymbol{\omega}-\boldsymbol{\omega} \cdot \boldsymbol{\sigma}$ denotes the Zaremba-Jaumann rate of Cauchy stress and $\boldsymbol{\omega} \stackrel{\text { def }}{=} \frac{1}{2}\left(\boldsymbol{\nabla} \boldsymbol{v}-(\boldsymbol{\nabla} \boldsymbol{v})^{\mathrm{T}}\right)$ is the vorticity tensor. In (51) we subtracted from the solid phase rate of deformation $\boldsymbol{d}^{\mathrm{sG}}$ the average volumetric strain rate $\dot{\varepsilon}^{\mathrm{sG}} \boldsymbol{I}$ of the compressible solid phase due to fluid phase pressure rate $\dot{p}^{\mathrm{fG}}$. The stress tensor $\sigma_{\mathrm{fr}}^{\mathrm{G}^{\prime \prime}}$ is responsible for all deformation of the solid phase, including the compression of grains. We should remark that the spatial gradient of solid phase volume fraction has been detected as fundamental in describing the quasistatic mechanical behavior of granular materials $[49,59,86,96]$, hence should be included in the list of arguments of $\boldsymbol{c}_{\mathrm{fr}}^{\mathrm{G}^{\prime}}$. However, for simplicity we assume here that this gradient is zero (homogeneous granular material).

It remains to specify a constitutive relation for the dynamic contribution $\boldsymbol{\sigma}_{\mathrm{vi}}^{\mathrm{G}^{\prime}}=\left(1-n^{\mathrm{G}}\right) \boldsymbol{\sigma}_{\mathrm{vi}}^{\mathrm{sG}}=\tilde{\boldsymbol{\sigma}}_{\mathrm{vi}}^{\mathrm{sG}}$ of the effective bulk stress in the dense grain-fluid mixture representing the saturated granular material. Different approaches are available, but most of them are restricted to particular flow conditions or to narrow ranges of material properties. In the present research, we adopt a simple model formulation suggested by Passman et al. [86] and further investigated in $[61,116]$. Its representation in rate form has been adopted in [31] to model silo discharge:

$$
\stackrel{\nabla}{\boldsymbol{\sigma}}_{\mathrm{vi}}^{\mathrm{G}^{\prime}} \stackrel{\text { def }}{=} 2 \mu^{\mathrm{G}^{\prime}} \boldsymbol{d}^{\mathrm{sG}}=\mu_{\mathrm{vol}}^{\mathrm{G}^{\prime}}\left(\operatorname{tr} \stackrel{\nabla}{\boldsymbol{\nabla}^{\mathrm{SG}}}\right) \boldsymbol{I}+2 \mu^{\mathrm{G}^{\prime}} \stackrel{\nabla}{\boldsymbol{d}_{\mathrm{dev}}^{\mathrm{sG}}}
$$

Such a form was also used in [56]. The first term on the right expresses the rate of stress change due to volume viscosity $\mu_{\text {vol }}^{\mathrm{G}^{\prime}}$. The dynamic shear viscosity $\mu^{\mathrm{G}^{\prime}}$ is generally a function of the void ratio (porosity) and shear rate. Relations have been proposed for different flow situations resp. flow geometries $[6,15,31,45,49$, $59,60,65,66,86]$. However, decision on which one is the most appropriate for the present class of problems requires further investigation.

\section{Interface Reconstruction and Propagation}

\section{$5.1 \quad$ Governing Equations}

One of the main features of the MMALE method is that material interfaces are not necessarily aligned with boundaries of the computational cells but may flow through the mesh (Fig. 2). The interface position needs to be known at each time step in order to achieve a reasonable accuracy of the overall method (Alg. 1). Different approaches are available in this context, as reviewed in $[27,62,100]$. The widely-used volume of fluid (VOF) methods [39,52, 58, 89, 93, 95, 118,119] do not track the interface directly, but instead track the fractional material volume in a mesh element by using an approximation to the interface. The interfaces 
are reconstructed $a b$ initio element by element from the solution data. Once the interface locations in each multi-material element have been determined, the material transport volumes across the element boundaries can be computed as truncation volumes. Finally, the fractional material volumes are integrated to a new time level to propagate the interface (Alg. 2). The actual calculation is largely geometrical in nature, as will be shown in the remainder of this paper.

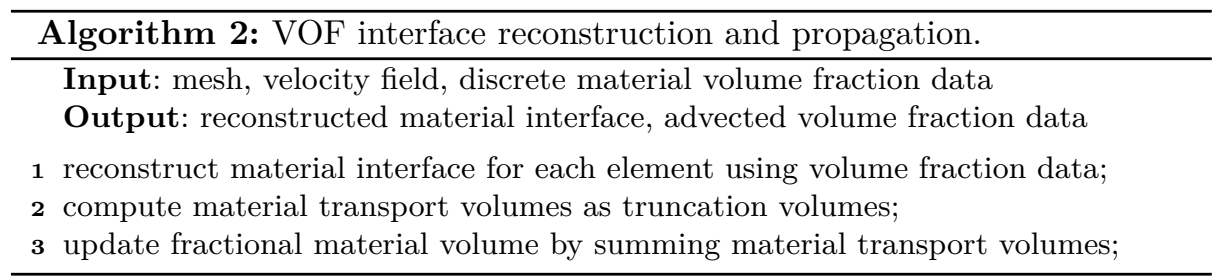

VOF methods are typically discussed with regard to a fixed, structured computational mesh of finite-difference type. The descriptions use volume coordinates and, in two dimensions, reduce the element to a unit square [24]. Concerning the present research, two points have to be considered. First, we seek to apply VOF methods to unstructured finite element meshes. This requires special data structures and coordinate systems which allow to resemble the classical descriptions. For example, in an unstructured quadrilateral mesh the parametric coordinates of the parent square element are equivalent to volume coordinates. Second, the mesh in MMALE methods is not fixed.

When using a Lagrangian-remap strategy, Alg. 2 is implemented by reconstructing the material interfaces in the Lagrangian elements prior to the remap; see also Alg. 1. The amount of transported material is usually defined as the regions swept out by the element facets during mesh relocation truncated by the interfaces. The interfaces that should be reconstructed in our MMALE method are not the microscopic interfaces between the grains and the pore fluid in the granular material, but the interfaces between the bulk materials on the macroscale (Fig. 3). For that reason, the material volume fraction $f^{k}$ has been introduced as a basic variable of our three-scale mixture theory. It naturally carries information based on which material interfaces can be reconstructed by using VOF methods.

Recall the situation and the basic notation introduced in Sect. 3.1. For reasons of simplification the present study is restricted to two-dimensional problems in Cartesian coordinates. Moreover, we consider the flow of only two materials $k \in\{1,2\}$, say, a light material and a dark material material, which are separated by a sharp material interface. The flow takes place in a time interval $[0, T] \subset \mathbb{R}$ and in a modeling domain $\mathcal{D} \subset \mathbb{R}^{2}$ of the ambient Euclidian space. A point in $\mathcal{D}$ is identified with its coordinate vector $\boldsymbol{x}=[x, y]^{\mathrm{T}} \in \mathbb{R}^{2}$. Velocities are assumed 
continuous at the interface, that is,

$$
\boldsymbol{v}_{1}=\boldsymbol{v}_{2} \equiv \overline{\boldsymbol{v}}
$$

No slip is currently taken into account. Hence, the interface velocity is the velocity normal to the interface [100]:

$$
\boldsymbol{v}_{\mathrm{I}}=v_{\mathrm{I}} \boldsymbol{n}_{\mathrm{I}}=\left(\overline{\boldsymbol{v}} \cdot \boldsymbol{n}_{\mathrm{I}}\right) \boldsymbol{n}_{\mathrm{I}},
$$

where $\boldsymbol{n}_{\mathrm{I}}$ is the field of unit normals on the interface, pointing outward the dark material.

Let $\chi$ be the material indicator function on the dark material in accordance with (18), then (19) and the application of (54) yield

$$
\frac{\partial \chi}{\partial t}+\overline{\boldsymbol{v}} \cdot \nabla \chi=0
$$

Taking the volume ${ }^{3}$ average resp. $\mathcal{H}$-average of this equation as defined in Sect. 3.1 and respecting the averaging rules $[41,42]$ results in

$$
\frac{\partial f}{\partial t}+\boldsymbol{v} \cdot \nabla f=0, \quad \text { or equivalently } \quad \dot{f}=0,
$$

where $f$ is the material volume fraction and $\boldsymbol{v}$ is an averaged velocity field, referred to as the (common) material velocity in what follows. Comparison with eq. (42) of the homogeneous equilibrium model derived in Sect. 3.3 indeed shows that the right sides of (56) are not zero in case of compressible materials. Therefore,

$$
\dot{f}=f\left(\frac{\langle K\rangle}{K}-1\right) \operatorname{div} \boldsymbol{v}
$$

is taken as the basic equation.

The material volume fraction is considered as the primary variable of VOF methods as it naturally carries information based on which material interfaces can be reconstructed. In fact, if $f=1$ then the zone is filled with dark material, and if $f=0$ the zone is filled with light material. A value $0<f<1$ indicates that the interface lies within that zone.

Following the derivations of Sect. 2, the ALE formulation of (57) is

$$
\frac{\partial \hat{f} J}{\partial t}+J \operatorname{div}(f \boldsymbol{c})=\frac{\langle K\rangle}{K} f J \operatorname{div} \boldsymbol{v}
$$

and the operator split associated with the Lagrange-remap strategy gives the two equations

$$
\begin{aligned}
\frac{\partial \hat{f} J}{\partial t} & =\frac{\langle K\rangle}{K} f J \operatorname{div} \boldsymbol{v}, \\
\frac{\partial \hat{f} J}{\partial t}+J \operatorname{div}(f \boldsymbol{c}) & =0 .
\end{aligned}
$$

\footnotetext{
${ }^{3}$ We use the term "volume" and "surface area" even though the present section is restricted to two-dimensional problems. In fact, area and length in two dimensions can be regarded as volume and surface area per unit depth in three dimensions.
} 
Again, (59) is equivalent to (57), but its integration in time is left unconsidered here. We just keep in mind that volume fraction might change during the Lagrangian step of the MMALE method. The second equation, (60), constitutes a conservation law and has to be solved during the remap step with respect to a given finite element mesh. This is outlined in the next sections.

\subsection{Basic Topological and Geometrical Functions}

The topological and geometrical information required for VOF interface reconstruction and propagation must be gathered from a given finite element mesh, consisting of $n_{\mathrm{el}}$ two-dimensional elements $\Omega_{e}$. The unique element number, $e$, is occasionally dropped. Each element is a simple polygon and represents a discrete portion of the reference domain moving and deforming in space. It is defined by nodes $I \in\left\{1, \ldots, n_{\mathrm{en}}\right\}$ and edges $\Gamma_{e, I}$ connecting the nodes $I$ and $I+1$. Nodes are numbered in counter-clockwise order of their occurrence along the element's perimeter, and the node $I=n_{\mathrm{en}}+1$ coincides with $I=1$. The global node number is denoted by $X$, and for each element $e$ and local node $I$ there is a unique number $X(I, e)$. The total number of nodal points in the mesh is $n_{\mathrm{np}}$. We assume for simplicity that global node numbering and element numbering is contiguous, so that $n_{\mathrm{np}}$ and $n_{\mathrm{el}}$ are equal to the largest node number and largest element number in the mesh, respectively. In other words, $X \in\left\{1, \ldots, n_{\mathrm{np}}\right\}$ and $e \in\left\{1, \ldots, n_{\mathrm{el}}\right\}$.

The numerical implementation of Alg. 2 requires topological information of the adjacent elements to an element, fundamental boolean set-theoretic operations applied to lines and polygons, also called clipping, as well as geometrical functions. In summary, the following functions are required:

1. Adjacent elements

2. Segment-segment intersection

3. Point-in-polygon test

4. Clipped polygon collection

5. Polygon volume

Adjacent Elements The transport resp. advection of material between elements requires the list of elements adjacent to each element. Data structures and the implementation of functions to determine these lists in unstructured quadrilateral meshes have been suggested in [28]. The basic information required in that reference is the two-dimensional connectivity array associated with the mesh. A modified approach is taken here.

In unstructured meshes working with dynamic structures like linked lists and pointer variables (available in Fortran 90 and above) is more practical than using fixed dimensional arrays. Beyond that, the current Fortran implementation adopts a somewhat object-oriented programming. Elements and nodes, for example, are derived data types which are comparable to those $\mathrm{C}++$ objects defined in [88]. Pointers to other data types resp. objects are stored within these data types, enabling the creation of a linked list with an arbitrary number of 
entries. By taking advantage of this option, the list of elements connected to a node is easily generated (Alg. 3).

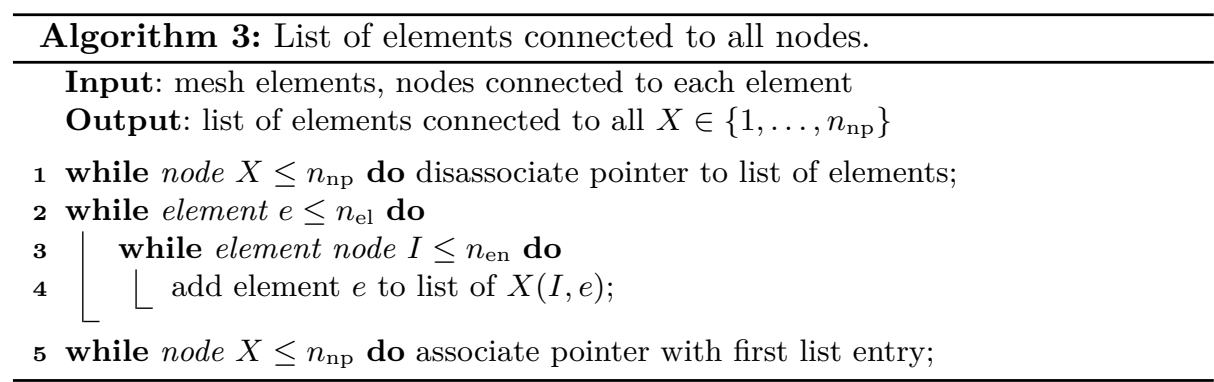

The element data type has an identifier $e \in\left\{1, \ldots, n_{\mathrm{el}}\right\}$, the element number, and points to an array of the local nodes $I \in\left\{1, \ldots, n_{\mathrm{en}}\right\}$ defining the element, and to an array of adjacent elements. Nodes are defined by a global node number $X \in\left\{1, \ldots, n_{\mathrm{np}}\right\}$ and a coordinate array. The global node number $X(I, e)$ assigned to the local node $I$ of an element $e$ has been traditionally defined as an entry of a mesh connectivity matrix. Because of their frequent use, the next node and the previous node of a particular node are also stored. The best way to do this is to store arrays containing the corresponding permutations of the local node numbers, $\operatorname{next}(I)=I+1$ and $\operatorname{prev}(I)=I-1$, respectively, in the definition of the element data type.

Once the list of elements connected to all nodes has been generated, the elements adjacent to an element can be determined according to the following procedure [28]. Edge $\Gamma_{I}$ of element $e$ is defined by node $I$ and the node counterclockwise from it, next $(I)=I+1$. The edge is shared by only two elements, the current element $e$ and the adjacent element, $\operatorname{adj}(e, I)$, sharing the edge with index $I$. A search on the lists of elements connected to the two nodes of the edge is carried out in order to determine the two elements in common. The element which is not the current element is the adjacent element (Alg. 4).

For the interface normal calculation outlined below, all the adjacent elements connected to all nodes of an element need to be known. Since the elements adjacent to an edge are known through Alg. 4, only the corner elements have to be determined. An easy way to generate this list is to copy the list resulting from Alg. 3 to the local nodes of all elements and to delete the current element and the elements adjacent to an edge.

Segment-Segment Intersection One basic function frequently used in VOF methods is the determination of the intersection point of line segments, i.e. between the interface and the element edges. A line segment, in contrast to infinite 


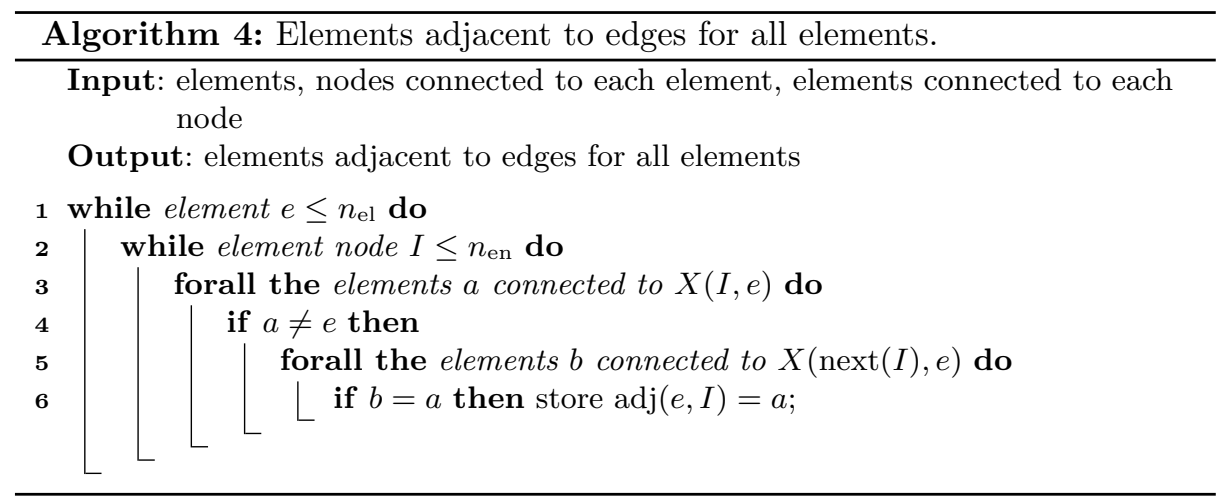

lines, has a finite length. Hence, there might be no intersection even if the segments are not parallel (invalid intersection). Several other cases generally have to be considered. We refer to [85, sect. 7.2] and [107] for further details and implementation.

Point-in-Polygon Test The point-in-polygon test is needed to determine whether a particular point or node lies inside a material zone. It is a basic operation frequently applied in computer graphics and other areas dealing with processing of geometrical data. One of the fastest solution strategies that requires only negligible amount of polygon data preprocessing is the crossing test [53]. A ray is shot from the test point commonly along an axis. Then, either the even/odd crossing number or winding number is computed to classify the point for arbitrary closed polygons $[2,44,53,85,101]$.

Clipped Polygon Collection VOF methods must frequently determine the partial material volumes (subzones) in the mesh elements or in the polygonal transport volumes across the element edges. Mathematically spoken, the subzone is the set-theoretic intersection of the domains enclosed by two polygons, or by one polygon and a half-plane. In computer graphics this is called polygon clipping [44,108]. The mesh represents a collection of clip polygons and the total material domain is represented by the subject polygon or half-plane.

In fact, not the polygon itself but only the vertices of its polygonal boundary line are stored. Polygon clipping has to determine the line segments belonging to the boundary of the subzone through intersection and has to merge these segments to close the boundary line; this decisive latter step is sometimes called "capping" in the literature. Hence, polygon clipping requires elaborate data structures and has to implement different boolean operations on polygons.

The data structures and clipping algorithm used in the present research have been developed in [50] and extended in [67] to handle degenerate cases. In both neither the clip nor the subject polygon needs to be convex, and they may have self-intersections. The each input and output polygon is efficiently represented 
as a doubly-linked edge list, referred to as the half-edge data structure. First, the segment-segment intersection points between the two input polygons are determined, then these are assigned specific flags to indicate relative orientation of the polygon edges, and finally the intersection points are merged into the data structure of the clipped subject polygons.

Polygon Volume The signed volume of a two-dimensional polygon defined by vertices $I \in\{1, \ldots, M\}$, with $M+1=1$, can be calculated from $[44,85,101,106]$

$$
V=\frac{1}{2} \sum_{I=1}^{M}\left(x_{I} y_{I+1}-x_{I+1} y_{I}\right)=\frac{1}{2} \sum_{I=1}^{M} x_{I}\left(y_{I+1}-y_{I-1}\right) .
$$

The first summation requires $2 M$ multiplications and $(2 M-1)$ additions, while the second summation requires only $M$ multiplications and $(2 M-1)$ additions [106]. Note that the signed volume is positive, i.e. $|V|=V$, if the vertices are placed in counter-clockwise order along the perimeter, otherwise it is negative.

It should be emphasized that the formula (61) can also be used to calculate the signed volume of a self-intersecting polygon. In this case the partial volumes adjacent to an intersection have opposite signs. For example, twisting a rectangle so that it looks like a figure 8 results in two triangular regions. Their volumes sum up to zero, which is the total signed volume of the twisted rectangle according to $(61)$.

\subsection{Interface Reconstruction}

Common state-of-the-art VOF methods approximate the interface in each multimaterial element by a straight line; see reviews in $[27,89,93]$. One of the earliest two-dimensional methods is due to Youngs [118], which forms a basis for the developments of the present research. Our implementation relies on that described in [95] because the original paper provides little detail of the interface reconstruction procedure. An alternative implementation is presented in [27].

A linear interface can be generally described by the Hesse normal form

$$
\boldsymbol{n} \cdot \boldsymbol{x}-d=0,
$$

in which $\boldsymbol{x}$ is an arbitrary point on the interface, $\boldsymbol{n}=\left[n^{x}, n^{y}\right]^{\mathrm{T}} \in \mathbb{R}^{2}$ is the unit normal on that interface (the index I has been dropped for notational brevity), and $d$ is the line constant representing the shortest distance between the interface and the origin. Most volume of fluid methods determine a linear reconstruction of the interface for each element in two steps: (i) estimate $\boldsymbol{n}$ and (ii) determine $d$ such that the volume fraction of the material lying behind the interface matches the known value.

Since we choose the normal to point outward of the material, (62) returns a positive number if $\boldsymbol{x}$ lies outside of the material. From the viewpoint of implementation, it proves convenient to introduce the gradient of the volume fraction, 
$\boldsymbol{m}=\left[m^{x}, m^{y}\right]^{\mathrm{T}}$, for which $\boldsymbol{n}=-\boldsymbol{m} /\|\boldsymbol{m}\|$. The slope of the interface, $s$, is related to the normal by $s=-m^{x} / m^{y}=-n^{x} / n^{y}$.

Youngs' method [118] has been developed for finite-difference type uniform meshes of square elements with edge lengths $\Delta x=\Delta y$; the original paper uses a mesh of unit squares. The interface slope is estimated based on the volume fraction data in the current element and its eight neighbors. Fig. 4 shows the notation for the element-centered volume fractions using the principal points of the compass. According to [95], the components of the volume fraction gradient in the current element of a uniform mesh can be approximated by the stencil

$$
\begin{aligned}
& m_{x}=\frac{1}{\Delta x}\left(f_{\mathrm{NE}}+2 f_{\mathrm{E}}+f_{\mathrm{SE}}-f_{\mathrm{NW}}-2 f_{\mathrm{W}}-f_{\mathrm{SW}}\right) \\
& m_{y}=\frac{1}{\Delta y}\left(f_{\mathrm{NE}}+2 f_{\mathrm{N}}+f_{\mathrm{NW}}-f_{\mathrm{SE}}-2 f_{\mathrm{S}}-f_{\mathrm{SW}}\right)
\end{aligned}
$$

The slope angle $\beta=\tan ^{-1} s=\tan ^{-1}\left(-m_{x} / m_{y}\right)$ lies in the range $-\pi / 2<$ $\beta<\pi / 2$. The stencil assumes that the elements are all unit volume. It would be not optimal, though practical to use the same stencil in structured quadrilateral meshes where the elements have different size. When using an gradient estimation according to (63), the interface reconstruction is only first-order accurate and linear interfaces are reproduced exactly only in certain isolated cases $[27,89,93$, 95].

Elements located at mesh boundaries require special treatment because one or more of the neighbors indicated in Fig. 4 might not exist. In this case the missing neighbors are substituted by so-called ghost elements, and the volume fraction of the current element is just copied to the ghost elements. The use of ghost elements also allows boundary conditions (e.g. inflow, outflow) to be handled efficiently in MMALE methods.

Once the slope or normal direction of the material interface is known, its location has to be determined by some procedure. The interface truncates the element domain, and the truncated volume behind the interface represents the partial material volume. Volume is conserved, i.e. the right location of the interface has been determined, if the partial volume divided by the element volume matches the given volume fraction data of that element. The matching can either be achieved through iteration of the distance parameter $d$, as done e.g. in [93], or by deriving an explicit expression that relates the truncated element volume to $d$ or to other parameters that locate the interface. The second approach has been pursued in $[27,52,119]$. We particularly follow the derivation of [95] again, implementing the original method [118].

If the element is a square, four principal cases of how the interface is located generally have to be considered (Fig. 5). In each case the interface intersects a particular pair of element edges under an angle

$$
\alpha=\tan ^{-1}\left(\frac{\Delta x}{\Delta y} \frac{-m_{x}}{m_{y}}\right), \quad \text { with } \quad 0 \leq \alpha \leq \pi / 2 .
$$




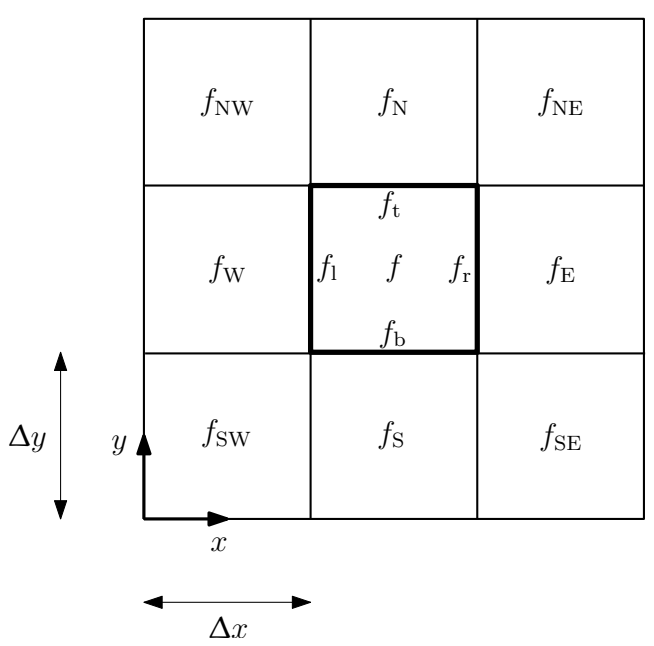

Fig. 4. Notation of adjacent element volume fractions and side fractions used for interface slope calculation.

The $3 \times 3$ block of elements eventually has to be rotated in such a way that $\alpha$ lies within the indicated range. The factor $\Delta x / \Delta y$ in the expression is only necessary if $m_{x}, m_{y}$ have been evaluated based on $\Delta x \neq \Delta y$. After the case has been determined by using $\alpha$ and the volume fraction $f$ of the element under consideration, the side fractions $f_{\mathrm{b}}, f_{\mathrm{r}}, f_{\mathrm{t}}$, and $f_{\mathrm{l}}$ at the bottom, right, top, and left edges, respectively, of the current element can be calculated (Fig. 4). The side fractions $f_{\sigma} \in[0,1], \sigma \in\{\mathrm{b}, \mathrm{r}, \mathrm{t}, \mathrm{l}\}$, are the fractions of the edges that lie within the material and uniquely determine the intersections of the interface with the element boundary. The logic to determine the case according to Fig. 5 and the calculation of the side fractions have been presented in $[95]^{4}$ and are summarized in Alg. 5.

As a simple example providing an analytical solution, we consider a linear interface on a fixed mesh of unit squares. The problem statement and mesh, including the element and global node numbers, is shown in Fig. 6. Application of the interface reconstruction algorithm described above to elements along the mesh boundary (element numbers 1,2, 6, etc.) requires a layer of ghost elements to complete the set of element neighbors for a real element. However, these ghost elements are not shown in Fig. 6 and in the following figures. The unit normal

\footnotetext{
${ }^{4}$ Tab. V in the original paper [95] has typos in the formulas for the side fractions for case IV, in which $C$ should be in fact $1-C$, where $C$ is the volume fraction. The correct formulas are in Alg. 5 .
} 


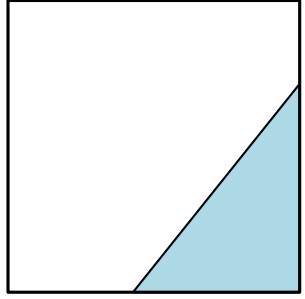

Case I

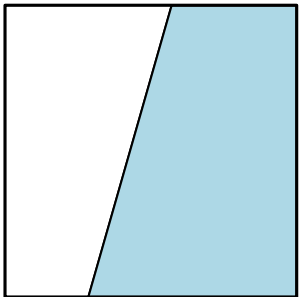

Case III

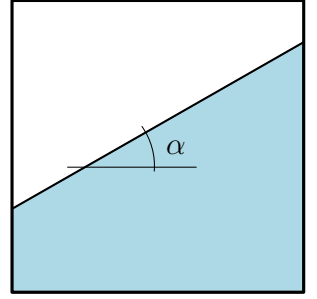

Case II

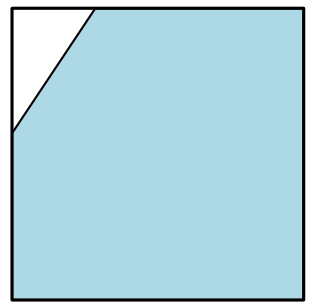

Case IV

Fig. 5. Four principal configurations of reconstructed interface for Youngs' method; after [95].

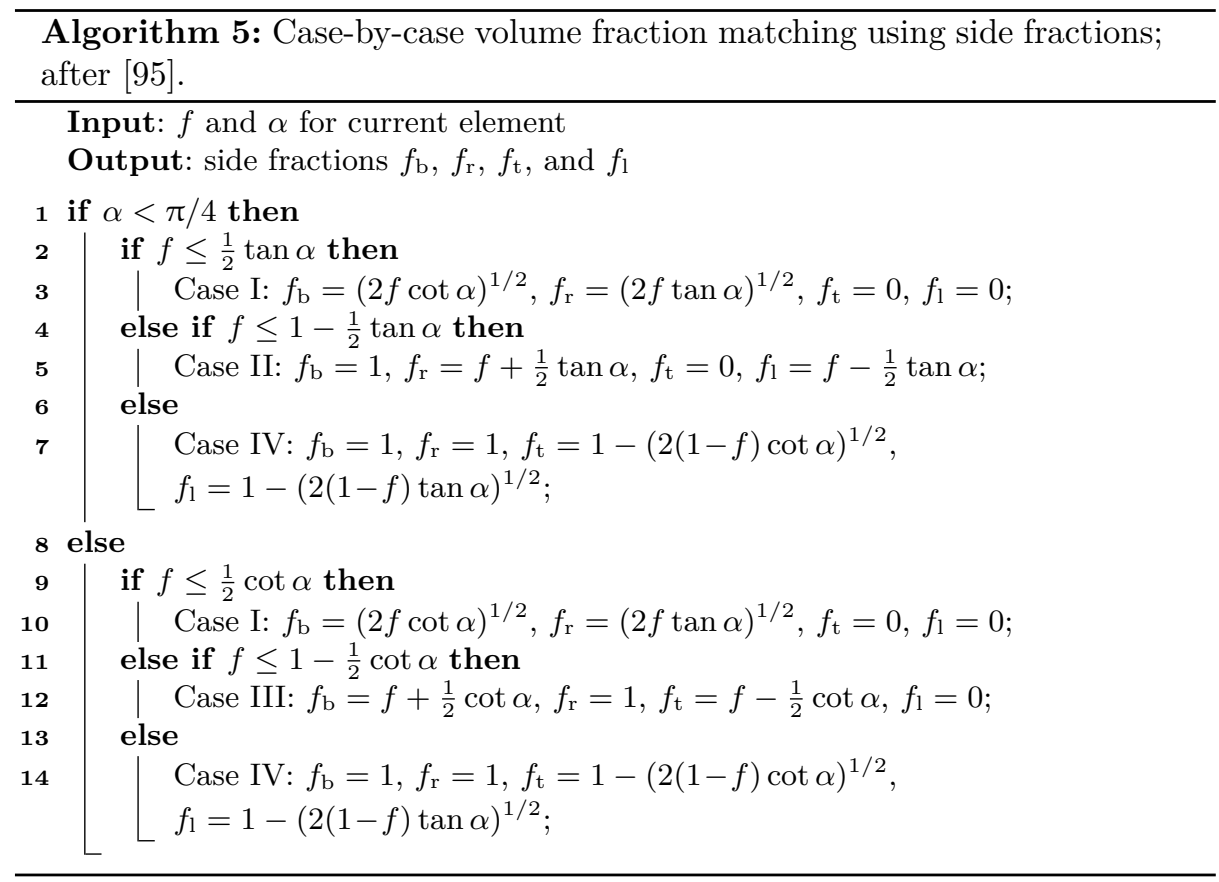


to the interface pointing outward the dark material is readily available through

$$
\boldsymbol{n}=\left\|\begin{array}{l}
5.0 \\
4.0
\end{array}\right\|^{-1}\left[\begin{array}{l}
5.0 \\
4.0
\end{array}\right]=\left[\begin{array}{l}
0.780871 \\
0.624697
\end{array}\right] \text {. }
$$

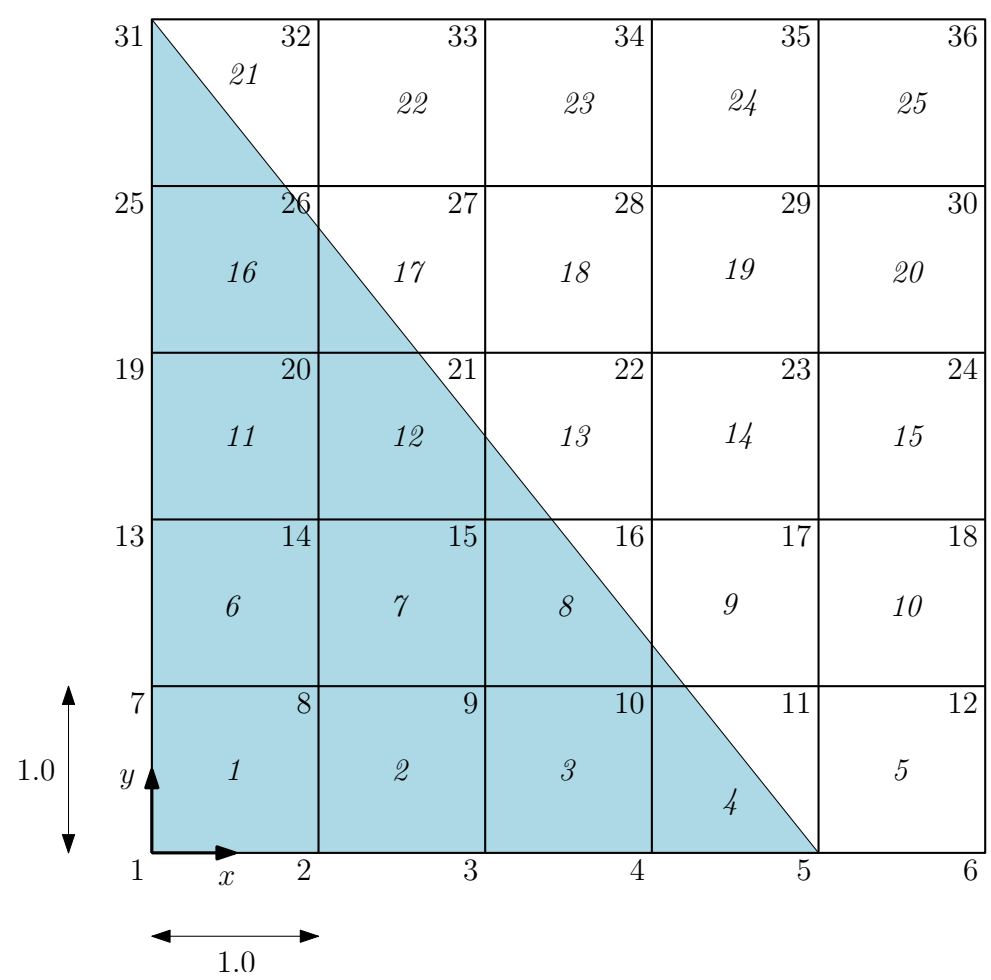

Fig. 6. Problem statement and mesh for linear interface reconstruction. The ghost elements along the mesh boundary are not shown.

The exact coordinates of the intersection points between the interface and the mesh in the initial configuration as well as the resulting volume fractions for all elements are plotted in Fig. 7. Since the volume of the elements is one, the volume fraction of an element is equal to the volume of the intersection between the dark material half-plane (triangular domain) and the element domain.

The interface is reconstructed by looping all real elements in the mesh and applying (63) and Alg. 5. The exact and reconstructed material interface using this method are compared in Fig. 8. Note that the reconstructed interface is not continuous across element boundaries because VOF methods work on a local level for reasons of efficiency. The element volume fractions are exactly matched 


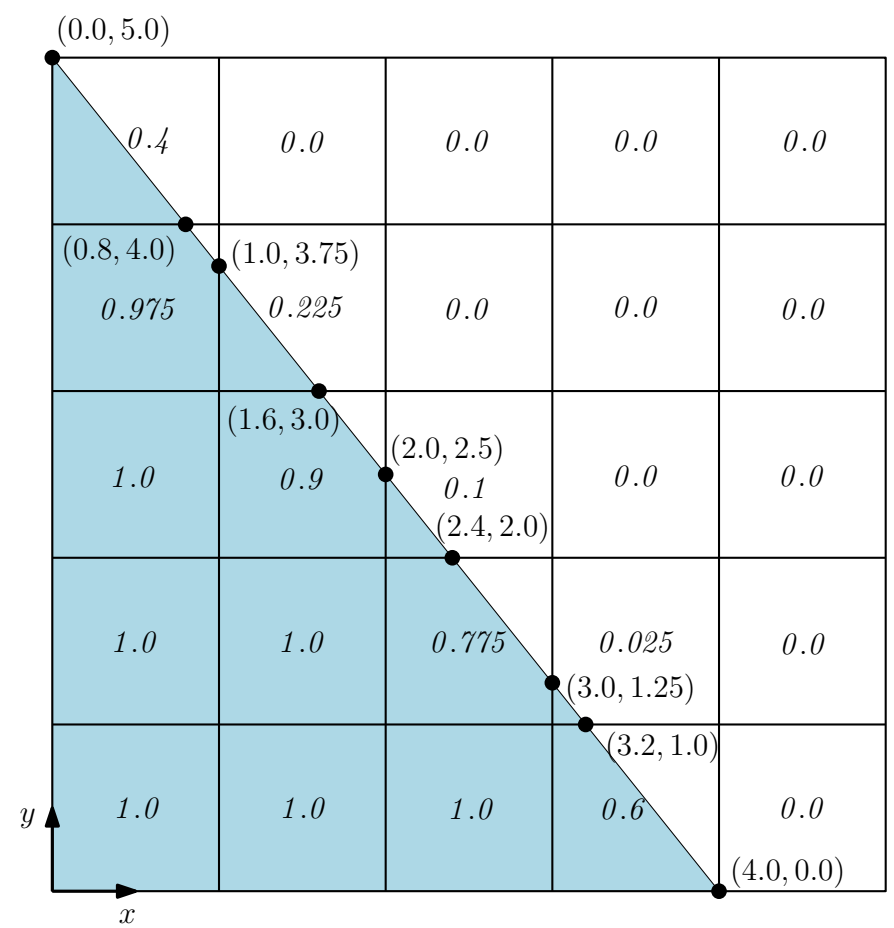

Fig. 7. Interface-mesh intersections (numbers in parentheses) and initial volume fractions (slanted numbers in the elements). 
by the reconstruction, but the interface slopes differ. The relative error of the slope angle, $E_{\beta}=\frac{2}{\pi} \| \beta_{\text {recon }}|-| \beta_{\text {exact }}||$, are listed in Tab. 5.3. It can be seen that the error is larger for elements located at mesh boundaries. This is because the volume fraction states in the ghost element neighbors are assigned the values of that element, which is only a rough approximation to the "true" state expected for an interface continuing beyond the mesh boundary.

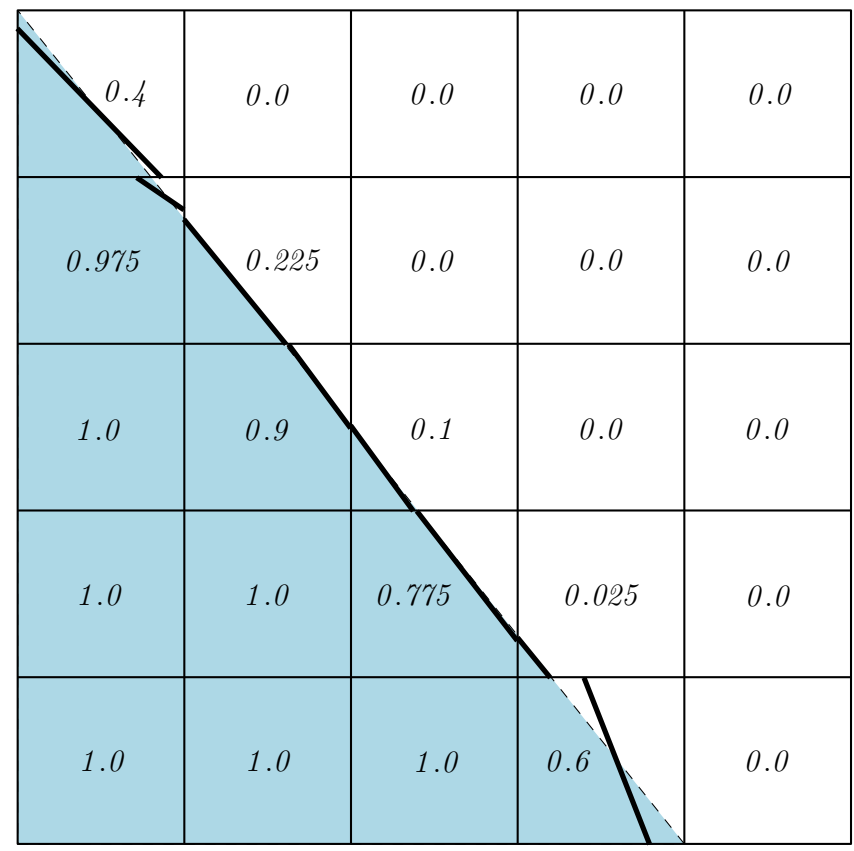

Fig. 8. Comparison of exact (dashed line) and reconstructed material interface (solid line) using Youngs' method $[95,118]$. Numbers in the elements denote the material volume fractions, which are exactly matched by the reconstruction.

\begin{tabular}{c|cccccccc} 
Element no. & 4 & 8 & 9 & 12 & 13 & 16 & 17 & 21 \\
\hline$E_{\beta}[\%]$ & 20.72 & 1.21 & 0.29 & 0.65 & 0.65 & 16.46 & 1.21 & 7.05
\end{tabular}

Table 1. Relative error of slope angle for elements with $0<f<1$. 


\subsection{Interface Propagation}

For a Lagrange-remap MMALE strategy, the interface is propagated through the mesh by transporting the fractional material volume across the element boundaries during the remap step. The time associated with the end of the Lagrangian step is denoted by $t^{-}$, while time associated with the end of remap step is $t^{+}$. The constant time increment is $\Delta t=t^{+}-t^{-}$; however, there is actually no physical time associated with it. On occasion, quantities related to the Lagrangian step and to the remap step are superscribed with - and +, respectively. Given the distorted geometry $\boldsymbol{x}^{-}$after the Lagrangian step and relocated geometry $\boldsymbol{x}^{+}$, the convective velocity is simply approximated by the finite difference formula $\boldsymbol{c}=\left(\boldsymbol{x}^{-}-\boldsymbol{x}^{+}\right) / \Delta t$. Data assumed to be given in the remap step includes both $\boldsymbol{x}^{-}$and $\boldsymbol{x}^{+}$as well as the material volume fraction distribution $f^{-}$constant in each element. If $\boldsymbol{x}^{+} \equiv \boldsymbol{x}_{0}$ is the original geometry at time $t=0$, then a Eulerian method is obtained [24].

Owing to (60), the remap takes the form of an advection problem subject to the initial condition $\left.f\right|_{t=t^{-}}=f^{-}$. Its numerical solution is done by a kind of finite volume method $[16,70]$. These methods are conservative by definition because they solve the integral form of (60). They work on a control volume tessellation which, in this paper, is assumed to coincide with the underlying finite element mesh. Therefore, the terms "control volume" and "element" can be used interchangeably.

The element boundary $\partial \Omega_{e}$ is piecewise oriented and $C^{1}$-continuous by definition. It is throughout assumed that the orientation of the boundary $\partial \Omega_{e}$ is compatible with the orientation of $\Omega_{e}$ such that the unit normals to $\partial \Omega_{e}$, denoted by $\boldsymbol{n}$, point outwards. In the context of finite volume methods, the control volume resp. element boundary is approximated by the set of edges $\Gamma_{e, I}$ of element $\Omega_{e}$, i.e.

$$
\partial \Omega_{e} \approx \bigcup_{I=1}^{n_{\mathrm{en}}} \Gamma_{e, I} \equiv \bigcup_{I} \Gamma_{e, I}
$$

Since overlaps and gaps of the mesh are precluded, there is a unique element $\Omega_{\operatorname{adj}(e, I)}$ adjacent to the edge $\Gamma_{e, I}$. If a vertex or edge of $\Omega_{e}$ is aligned with the boundary of the computational domain, a ghost element $\Omega_{j}$ is added such that the intersection $\Omega_{e} \cap \Omega_{j}$ is non-zero.

Based on the previous definitions, we seek an approximate solution to the integral form of the ALE conservation law (60) with respect to each control volume:

$$
\frac{\mathrm{d}}{\mathrm{d} t} \int_{\Omega_{e}} f \mathrm{~d} v+\sum_{I} \int_{\Gamma_{e, I}} f \boldsymbol{c} \cdot \boldsymbol{n} \mathrm{d} a=0, \quad \text { subject to }\left.\quad f\right|_{t=t^{-}}=f^{-} .
$$

The product $f \boldsymbol{c}$ in the second term on the left side is the convective flux of the field $f$, and $\mathrm{d} a$ is the surface area density on $\mathbb{R}^{2}$ (representing the differential arc length). In solving the problem defined through (67), the method approximates 
the evolution of the element average

$$
f_{e}=\frac{1}{V_{e}} \int_{\Omega_{e}} f \mathrm{~d} v, \quad \text { with } \quad V_{e}=\int_{\Omega_{e}} 1 \mathrm{~d} v>0 \quad \text { and } \quad e \in\left\{1, \ldots, n_{\mathrm{el}}\right\},
$$

over the pseudo time interval $\left[t^{-}, t^{+}\right]$. The averaged convective volume flux across a moving and deforming element edge $\Gamma_{e, I}$ is defined by

$$
F_{e, I}^{V}=\frac{1}{S_{e, I}} \int_{\Gamma_{e, I}} \boldsymbol{c} \cdot \boldsymbol{n} \mathrm{d} a, \quad \text { where } \quad S_{e, I}=\int_{\Gamma_{e, I}} 1 \mathrm{~d} a>0 .
$$

Note that volume flux is positive if material leaves the moving element through the boundary. The measure $S_{e, I}$ is the surface area of the edge $\Gamma_{e, I}$.

For every pair of adjacent elements $\left(\Omega_{e}, \Omega_{\operatorname{adj}(e, I)}\right)$, the true averaged convective flux of the field $f$ across the edge $\Gamma_{e, I}=\Omega_{e} \cap \Omega_{\operatorname{adj}(e, I)}$ is replaced by a numerical averaged convective flux

$$
F_{e, I}^{f} \approx \frac{1}{S_{e, I}} \int_{\Gamma_{e, I}} f \boldsymbol{c} \cdot \boldsymbol{n} \mathrm{d} a
$$

We require $F_{e, I}^{f=1}=F_{e, I}^{V}$ for reasons of consistency. This is most easily achieved by defining

$$
F_{e, I}^{f}=\eta_{e, I}^{\star} F_{e, I}^{V},
$$

where $\eta_{e, I}^{\star}$ represents a particular volume fraction at the element edge $\Gamma_{e, I}$ determined by an appropriate volume of fluid algorithm.

Now, substitution of (68)-(71) into (67) yields the space-discrete problem

$$
\frac{\mathrm{d}}{\mathrm{d} t}\left(f_{e} V_{e}\right)+\sum_{I} \eta_{e, I}^{\star} S_{e, I} F_{e, I}^{V}=0
$$

for all $e \in\left\{1, \ldots, n_{\mathrm{el}}\right\}$ and subject to $\left.f\right|_{t=t^{-}}=f^{-}$. Discretization in time for solid mechanical applications is commonly done by first-order accurate explicit integration methods. In particular, application of the forward Euler method to (72) results in the advection algorithm

$$
f_{e}^{+}=\frac{f_{e}^{-} V_{e}^{-}-\sum_{I} \Delta V_{e, I}^{f}}{V_{e}^{+}}, \quad \text { with } \quad V_{e}^{+}=V_{e}^{-}-\sum_{I} \Delta V_{e, I}
$$

$\Delta V_{e, I}=S_{e, I} F_{e, I}^{V} \Delta t$, and $\Delta V_{e, I}^{f}=\eta_{e, I}^{\star} \Delta V_{e, I}$. Here $V_{e}^{-}$is the volume of the deformed element in the Lagrangian mesh, $V_{e}^{+}$is the element volume in the relocated mesh, $\Delta V_{e, I}$ is the total transported volume across the edge $\Gamma_{e, I}$ between $\Omega_{e}$ and the element $\Omega_{\operatorname{adj}(e, I)}$ adjacent to $\Gamma_{e, I}$, and $\Delta V_{e, I}^{f}$ represents the material transport volume across that edge. $\Delta V_{e, I}$ is defined positive if the nodes defining the edge are moved further into the element's region, that is, if the transport volume is leaving the element. 
Explicit advection algorithms, like (73), give rise to stability issues. Stability of a numerical algorithm ensures that the local errors introduced by the approximate solution are not amplified and that the global error produced by the algorithm after several time steps is bounded. A necessary stability condition for any transport algorithm is the CFL condition [46]

$$
0 \leq \quad C o=\frac{\|c \Delta t\|}{h} \leq 1
$$

Here $h>0$ is a characteristic element length and Co is called the CFL number. The CFL condition phrases that a material particle must not pass an element within one step. Concerning the remap step of the MMALE method, the condition (74) constrains the difference of nodal positions before and after the remap through $\left\|\boldsymbol{x}^{-}-\boldsymbol{x}^{+}\right\| \leq h$.

The amount of material transported across an element edge is represented by the flux term $\Delta V_{e, I}^{f}$ in the generic advection algorithm (73). This flux term can be calculated without interface reconstruction once a particular advection scheme has been assigned [95]. However, it is more common, and yet more accurate, to incorporate the geometry of the interface and to compute the material transport volumes as truncation volumes [27,39,46,52,88,93,118]. In this second approach, the total transport volume across an element edge is the signed volume swept out by that edge between its two configurations after the Lagrangian step and after the remap. The material transport volume is simply the set-theoretic intersection of the total transport volume and the material domain on the left of the interface (for a normal pointing outwards resp. to the right). However, the actual implementation is more complicated.

The pseudocode of a suitable implementation of the volume fraction update in the remap step using (73) is provided in Alg. 6, and it is based on a procedure outlined in [28]. Note that a negative total transport volume is set to zero, and a volume subtracted from element $e$ is added to element $\operatorname{adj}(e, I)$ adjacent to edge $I$ to avoid double counts. Moreover, the value of the total and material volume update is calculated only once if the total transport volume is positive. This eliminates half of the remap operations.

\section{Conclusions and Outlook}

An innovative theoretical and numerical framework has been presented which accounts for the large deformations and evolving material interfaces as well as for the complex coupled material behavior of saturated granular material present in geomechanical multi-material flow. In order to model the large-scale motions and interactions of materials in such flow situations, an arbitrary LagrangianEulerian method has been developed in which multi-material elements carry the information of the material interfaces and free surfaces (MMALE method).

The states of the individual materials in multi-material elements are derived from a practical three-scale mixture theory and a homogeneous equilibrium 


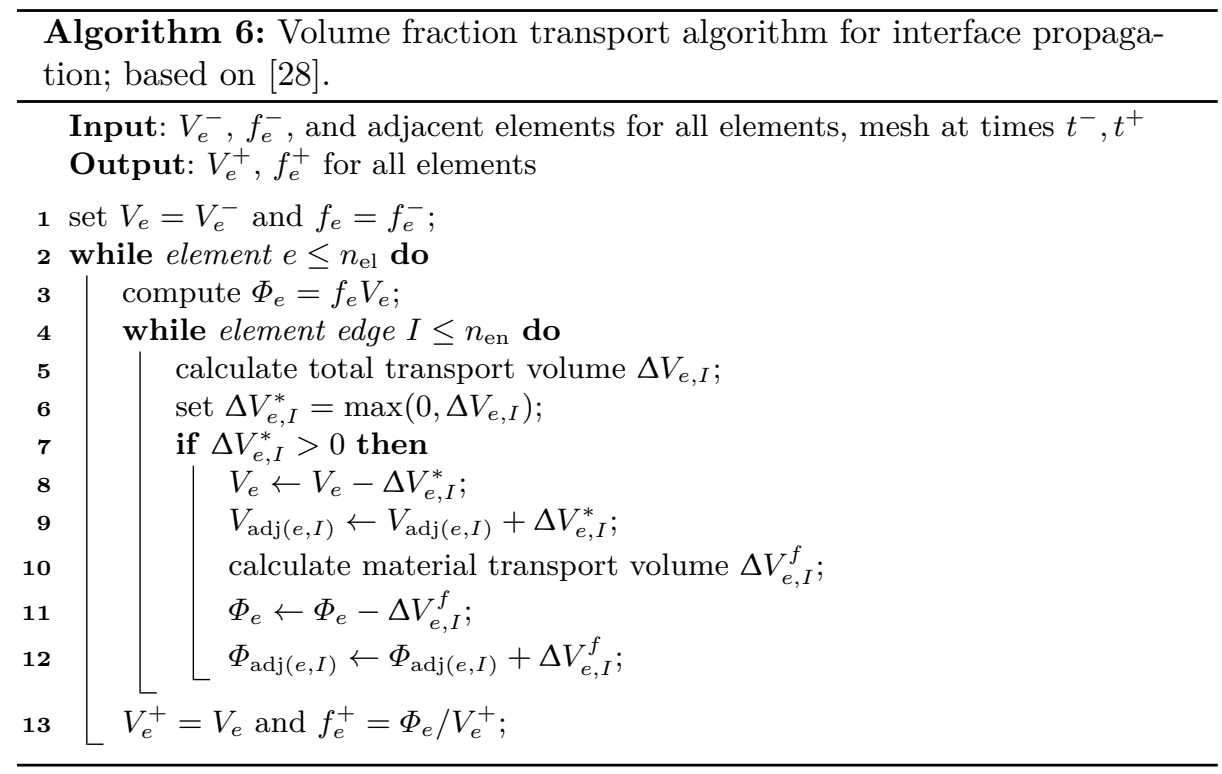

model developed during this research. In contrast to common two-scale theories, the proposed three-scale approach is able to incorporate both the evolution of bulk material interfaces as well as the two-phase phenomena associated with saturated granular material. The essential closure relations are specified with respect to the macroscale. We have considered an example flow consisting of a bulk solid, a bulk fluid, and a fluid-saturated granular material with compressible constituents. The mixture approach allows for the use of phenomenological constitutive models describing granular material response for the full frictionalcollisional flow regime. Accordingly, the apparent stress tensors in the dense grain-fluid mixture have been split into rate-independent and rate-dependent parts.

In the MMALE method, material interfaces are reconstructed $a b$ initio and propagated through the computational mesh by using a volume of fluid (VOF) approach. It has been shown that the governing equations are consistent with the developed three-scale mixture theory and with the Lagrange-remap MMALE strategy. The geometrical and topological functions required for the VOF method have been outlined and efficient algorithms have been presented. The basic features were tested by using a simple example for which an analytical solution is available. Future work will focus on the extension to unstructured triangle meshes, the implementation into the MMALE method, and on the solution of full-scale large deformation problems in geomechanics and geotechnical engineering, including geotechnical installation processes.

Acknowledgments. The presented work was carried out under the financial support from the German Research Foundation (DFG; Grant SA 310/26-2) as 
part of the DFG Research Unit FOR 1136, which is gratefully acknowledged. The authors would like to thank their colleagues in this research unit for collaboration and continuously discussing our work. Special thanks go to Prof. David J. Benson and the Department of Structural Engineering at the University of California, San Diego (UCSD) for the opportunity to undertake collaborative research on MMALE methods.

\section{Nomenclature}

\section{Operators and Special Notations}

$\cdot,:, \otimes \quad$ single contraction, double contraction, tensor product

$\cup, \cap, \backslash$ union, intersection, and difference of sets

$|\cdot|,|| \cdot||$ absolute value, Euclidean length

$\langle\cdot\rangle \quad$ spatial average

$(\cdot),(\cdot)^{\alpha k}$ material time derivative, of an $\alpha k$-related field

$($.$) referential, ALE description$

$(.)^{\alpha k} \quad \alpha k$-intrinsic average

$(\cdot)^{[\alpha k]} \quad$ limit value at $\alpha k$-boundary

$(\cdot) \quad$ Zaremba-Jaumann rate

$\boldsymbol{\nabla}(\cdot) \quad$ covariant derivative, gradient

$\partial(\cdot) \quad$ boundary

$\operatorname{div}(\cdot) \quad$ divergence

$\operatorname{tr}(\cdot) \quad$ trace of a second-order tensor

\section{Superscripts and Subscripts}

$\begin{array}{ll}-,+ & \text { associated with Lagrangian step, remap step } \\ \text { adj } & \text { adjacent } \\ \text { dev } & \text { deviator of a second-order tensor } \\ \text { dr } & \text { drained } \\ \mathrm{f}, \mathrm{fG}, \mathrm{f} k & \text { fluid phase, in granular material, in } k \text {-material } \\ \mathrm{fr} & \text { frictional (rate-independent) contribution } \\ \mathrm{F} & \text { bulk fluid; } \mathrm{F} \equiv \mathrm{fF} \\ \mathrm{G} & \text { fluid-saturated granular material } \\ \mathrm{G}^{\prime} & \text { related to effective stress in granular material }\end{array}$


$k \quad k$-material; $k \in\{\mathrm{S}, \mathrm{F}, \mathrm{G}\}=\{1, \ldots, M\}$

$\mathrm{s}, \mathrm{sG}, \mathrm{s} k$ solid phase, in granular material, in $k$-material

$\mathrm{S} \quad$ bulk solid; $\mathrm{S} \equiv \mathrm{sS}$

$\mathrm{T}$ transpose of a tensor

uj unjacketed

vi viscous (rate-dependent) contribution

vol volume

$\alpha \quad \alpha$-phase; $\alpha \in\{\mathrm{s}, \mathrm{f}\}=\{1, \ldots, N\}$

$\alpha k \quad \alpha$-phase in $k$-material; $\alpha k \in\{\mathrm{S}, \mathrm{F}, \mathrm{sG}, \mathrm{fG}\}$

$\alpha \beta k l \quad$ interface between $\alpha k$ and $\beta l$

\section{Latin Symbols}

$\begin{array}{ll}\boldsymbol{b}, \boldsymbol{b}^{\alpha k},\langle\boldsymbol{b}\rangle & \text { body force per unit mass } \\ \boldsymbol{c} & \text { convective velocity } \\ \boldsymbol{c}_{\mathrm{fr}}^{\mathrm{G}^{\prime}} & \text { fourth-order material tangent tensor } \\ C o & \text { CFL number } \\ \boldsymbol{d}, \boldsymbol{d}^{\alpha k} & \text { spatial rate of deformation } \\ \mathrm{d} a, \mathrm{~d} v & \text { surface area density, volume density } \\ d & \text { line constant } \\ \mathcal{D} & \text { modeling domain in the ambient space } \\ e & \text { element } \\ e^{\mathrm{G}} & \text { void ratio } \\ f, f^{k}, f^{\alpha k}, f_{e} & \text { volume fractions, of } k, \alpha k, \text { in element } \\ f_{\mathrm{b}}, f_{\mathrm{r}}, f_{\mathrm{t}}, f_{\mathrm{l}} & \text { side fractions } \\ F, F_{e, I}^{V}, F_{e, I}^{f} & \text { convective flux, of } V_{e}, f_{e} \text { across } \Gamma_{e, I} \\ \boldsymbol{h}^{\mathrm{G}^{\prime}} & \text { set of material state variables } \\ H, H^{k}, H^{\alpha k} & \text { volume measures of } \mathcal{H}, \mathcal{H}^{k}, \mathcal{H}^{\alpha k} \\ \mathcal{H} & \text { representative volume element }(\mathrm{RVE}) \\ \mathcal{H}^{k}, \mathcal{H}^{\alpha k} & \text { portions of } k, \alpha k \text { in } \mathcal{H} \\ I & \text { local node, vertex, edge } \\ \boldsymbol{I} & \text { second-order unit tensor } \\ J & \text { Jacobian } \\ K, K^{k}, K^{\alpha k},\langle K\rangle & \text { bulk modulus } \\ l_{\text {micro }}, l_{\text {meso }}, l_{\text {macro }} & \text { microscale, mesoscale, macroscale }\end{array}$


$m$

M

$\mathcal{M}^{k}$

$n, n^{k}, n^{\mathrm{G}}$

$n_{e l}, n_{e n}, n_{n p}$

$\boldsymbol{n}, \boldsymbol{n}^{\alpha k}$

$N$

$p^{\alpha k}, p^{k},\langle p\rangle$

$p^{\mathrm{G}^{\prime}}$

$\mathcal{P}^{\alpha}$

$q, q^{k}, q^{\alpha k}$

$\mathbb{R}, \mathbb{R}^{2}, \mathbb{R}^{3}$

$s$

$\boldsymbol{s}^{k}, \boldsymbol{s}^{\alpha k},\langle\boldsymbol{s}\rangle$

$S_{e, I}$

$t, t^{-}, t^{+}$

$\boldsymbol{v}, \boldsymbol{v}^{k}, \boldsymbol{v}^{\alpha k},\langle\boldsymbol{v}\rangle$

$\boldsymbol{v}_{\mathrm{I}}, \boldsymbol{v}_{\mathrm{I}}^{\alpha k}, \boldsymbol{v}_{\mathrm{I}}^{\mathrm{m}}$

$V_{e}$

$\boldsymbol{w}$

$x, \boldsymbol{x}$

$x, y$

$X$ volume fraction gradient

number of materials in the mixture

$k$-material domain in $\mathcal{D}$

fluid fraction, porosity

number of elements, element nodes, nodal points

outward normals on interface

number of phases in the mixture

pressure

mean effective stress

$\alpha$-phase domain in $\mathcal{D}$

generic spatial field

real numbers, ambient Euclidian space

slope

extra stress

surface area of $\Gamma_{e, I}$

time

spatial velocity

interface velocity

element volume

mesh velocity

point in the ambient space

spatial coordinate directions; $\boldsymbol{x}=[x, y]^{\mathrm{T}}$

global node number

\section{Greek Symbols}

$\begin{array}{ll}\alpha, \beta & \text { slope angle } \\ \Gamma_{I}, \Gamma_{e, I} & \text { edge, element edge } \\ \Gamma^{\alpha k} & \text { rate of momentum supply due to drag via } \partial \mathcal{H}^{\alpha k} \\ \delta_{\mathrm{I}}^{\alpha k} & \text { Dirac delta picking out the } \alpha k \text {-interface } \\ \Delta V_{e, I}, \Delta V_{e, I}^{f} & \text { total transport volume, material transport volume } \\ \Delta t & \text { time increment } \\ \Delta x, \Delta y & \text { element dimensions } \\ \dot{\varepsilon}^{\mathrm{sG}} & \text { solid phase volumetric strain rate due to } \dot{p}^{\mathrm{fG}} \\ \zeta^{\mathrm{G}} & \text { Biot-Willis coefficient }\end{array}$




$\begin{array}{ll}\eta_{e, I}^{\star} & \text { volume fraction at } \Gamma_{e, I} \\ \lambda^{\mathrm{F}} & \text { second viscosity coefficient } \\ \Lambda^{\alpha k} & \text { rate of mass supply via } \partial \mathcal{H}^{\alpha k} \\ \mu^{\mathrm{F}}, \mu^{\mathrm{fF}}, \mu^{\mathrm{G}^{\prime}} & \text { dynamic shear viscosity } \\ \mu_{\mathrm{vol}}^{\mathrm{G}^{\prime}} & \text { volume viscosity } \\ \pi^{\alpha k} & \text { volume fraction of } \alpha \text { with respect to } \mathcal{H}^{k} \\ \rho, \rho^{k}, \rho^{\alpha k},\langle\rho\rangle & \text { spatial mass density } \\ \boldsymbol{\sigma}, \boldsymbol{\sigma}^{k}, \boldsymbol{\sigma}^{\alpha k},\langle\boldsymbol{\sigma}\rangle & \text { (Cauchy) stress } \\ \boldsymbol{\sigma}^{\mathrm{G}^{\prime}} & \text { effective stress } \\ \chi^{k}, \chi^{\alpha}, \chi^{\alpha k} & \text { indicator function } \\ \boldsymbol{\omega} & \text { vorticity tensor } \\ \Omega_{e} & \text { element domain, control volume }\end{array}$

\section{References}

1. S. Achanta, J. H. Cushman, and M. R. Okos. On Multicomponent, Multiphase Thermomechanics with Interfaces. International Journal of Engineering Science, 32(11):1717-1738, 1994.

2. D. Alciatore and R. Miranda. A Winding Number and Point-In-Polygon Algorithm. Glaxo Virtual Anatomy Project Research Report, Department of Mechanical Engineering, Colorado State University, 1995.

3. C. Ancey, P. Coussot, and P. Evesque. A Theoretical Framework for Granular Suspensions in a Steady Simple Shear Flow. Journal of Rheology, 43(6):1673-1699, 1999.

4. C. Ancey and P. Evesque. Frictional-Collisional Regime for Granular Suspension Flows Down an Inclined Channel. Physical Review E, 62(6):8349-8360, 2000.

5. C. Ancey. Dry Granular Flows Down an Inclined Channel: Experimental Investigations on the Frictional-Collisional Regime. Physical Review E, 65(011304), 2001.

6. C. Ancey. Plasticity and Geophysical Flows: A Review. Journal of Non-Newtonian Fluid Mechanics, 142:4-35, 2007.

7. K. G. Anderson and R. Jackson. A Comparison of the Solutions of Some Proposed Equations of Motion of Granular Materials for Fully Developed Flow Down Inclined Planes. Industrial \& Engineering Chemistry Fundamentals, 241:145-168, 1992.

8. D. Aubram. Differential Geometry Applied to Continuum Mechanics. Number 44 in Veröffentlichungen des Grundbauinstitutes der Technischen Universität Berlin. Shaker Verlag, Aachen, 2009. http://opus.kobv.de/tuberlin/volltexte/2009/ 2270/

9. D. Aubram. An Arbitrary Lagrangian-Eulerian Method for Penetration into Sand at Finite Deformation. Number 62 in Veröffentlichungen des Grundbauinstitutes der Technischen Universität Berlin. Shaker Verlag, Aachen, 2013. http://opus4.kobv . de/opus4-tuberlin/frontdoor/index/index/docId/4755

10. D. Aubram. Development and Experimental Validation of an Arbitrary Lagrangian-Eulerian (ALE) Method for Soil Mechanics. Geotechnik, 38(3), 2015 (in press). 
11. D. Aubram. Homogeneous Equilibrium Model for Three-Scale Geomechanical Multi-Material Flow (in preparation).

12. D. Aubram, F. Rackwitz, and S. A. Savidis. An ALE Finite Element Method for Cohesionless Soil at Large Strains: Computational Aspects and Applications. In T. Benz and S. Nordal (eds.): Proceedings 7th European Conference on Numerical Methods in Geotechnical Engineering (NUMGE), pages 245-250. CRC Press, Boca Raton, 2010.

13. D. Aubram, F. Rackwitz, P. Wriggers, and S. A. Savidis. An ALE Method for Penetration into Sand Utilizing Optimization-Based Mesh Motion. Computers and Geotechnics, 65:241-249, 2015. http://dx.doi.org/10.1016/j.compgeo.2014.12. 012

14. D. Aubram, F. Rackwitz, and S. A. Savidis. Vibro-Injection Pile Installation in Sand: Part I-Interpretation as Multi-Material Flow. In Th. Triantyfyllidis (ed): Holistic Simulation of Geotechnical Installation Processes - Numerical and Physical Modelling, pages 73-102. Springer International Publishing, 2015. http://dx.doi.org/10.1007/978-3-319-18170-7_5

15. R. A. Bagnold. Experiments on a Gravity-Free Dispersion of Large Solid Spheres in a Newtonian Fluid Under Shear. Proceedings of the Royal Society of London. Series A, 225:49-63, 1954.

16. T. Barth and M. Ohlberger. Finite Volume Methods: Foundation and Analysis. Volume 1 of Encyclopedia of Computational Mechanics, chapter 15. John Wiley \& Sons, Ltd., 2004.

17. E. Bauer. Calibration of a Comprehensive Constitutive Equation for Granular Materials. Soils and Foundations, 36(1):13-26, 1996.

18. A. Bedford and D. S. Drumheller. Theories of Immiscible and Structured Mixtures. International Journal of Engineering Science, 21(8):863-960, 1983.

19. T. Belytschko, W. K. Liu, and D. Moran. Nonlinear Finite Elements for Continua and Structures. John Wiley \& Sons, Ltd., 2000.

20. L. S. Bennethum. Compressibility Moduli for Porous Materials Incorporating Volume Fraction. Journal of Engineering Mechanics, 132:1205-1214, 2006.

21. L. S. Bennethum and J. H. Cushman. Multiscale, Hybrid Mixture Theory for Swelling Systems-I. Balance Laws. International Journal of Engineering Science, 34(2):125-145, 1996.

22. L. S. Bennethum and J. H. Cushman. Multiscale, Hybrid Mixture Theory for Swelling Systems-II. Constitutive Theory. International Journal of Engineering Science, 34(2):147-169, 1996.

23. L. S. Bennethum and T. Weinstein. Three Pressures in Porous Media. Transport in Porous Media, 54:1-34, 2004.

24. D. J. Benson. Computational Methods in Lagrangian and Eulerian Hydrocodes. Computer Methods in Applied Mechanics and Engineering, 99:235-394, 1992.

25. D. J. Benson. A Multi-Material Eulerian Formulation for the Efficient Solution of Impact and Penetration Problems. Computational Mechanics, 15:558-571, 1995.

26. D. J. Benson. An Implicit Multi-Material Eulerian Formulation. International Journal for Numerical Methods in Engineering, 48:475-499, 2000.

27. D. J. Benson. Volume of Fluid Interface Reconstruction Methods for MultiMaterial Problems. Applied Mechanics Reviews, 55(2):151-165, 2002.

28. D. J. Benson. Momentum Advection On Unstructured Staggered Quadrilateral Meshes. International Journal for Numerical Methods in Engineering, 75:1549-1580, 2008.

29. M. A. Biot. General Theory of Three-Dimensional Consolidation. Journal of Applied Physics, 12:155-164, 1941. 
30. M. A. Biot and D. G. Willis. The Elastic Coefficients of the Theory of Consolidation. Journal of Applied Mechanics, 24:594-601, 1957.

31. J. U. Böhrnsen, H. Antes, M. Ostendorf, and J. Schwedes. Silo Discharge: Measurement and Simulation of Dynamic Behavior in Bulk Solids. Chemical Engineering \&) Technology, 27:71-76, 2004.

32. J. A. Bouré and J. M. Delhaye. General Equations and Two-Phase Flow Modeling Section 1.2 in G. Hetsroni (ed.): Handbook of Multiphase Systems. Hemisphere Publishing Corporation, 1982.

33. J. A. Bouré. Two-Phase Flow Models: The Closure Issue. Multiphase Science and Technology, 3(1-4):3-30, 1987.

34. R. M. Bowen. Compressible Porous Media Models by Use of the Theory of Mixtures. International Journal of Engineering Science, 20(6):697-735, 1982.

35. P. Colella, H. M. Glaz, and R. E. Ferguson. Multifluid Algorithms for Eulerian Finite Difference Methods. 1997 (unpublished manuscript).

36. P. Coussot and C. Ancey. Rheophysical Classification of Concentrated Suspensions and Granular Pastes. Physical Review E, 59(4):4445-4457, 1999.

37. J. H. Cushman, L. S. Bennethum, and B. X. Hu. A Primer on Upscaling Tools for Porous Media. Advances in Water Resources, 25:1043-1067, 2002.

38. B. M. Das. Advanced Soil Mechanics. Taylor \& Francis, USA, 3rd ed., 2008.

39. R. B. DeBar. Fundamentals of the KRAKEN Code. Technical Report UCID-17366, Lawrence Livermore Laboratory, Livermore, USA, 1974.

40. R. De Boer. Theory of Porous Media. Springer-Verlag, Berlin, 2000.

41. D. A. Drew. Mathematical Modeling of Two-Phase Flow. Annual Review of Fluid Mechanics, 15:261-291, 1983.

42. D. A. Drew and S. L. Passman. Theory of Multicomponent Fluids. Springer, New York, 1999.

43. D. S. Drumheller. A Theory for Dynamic Compaction of Wet Porous Solids. International Journal of Solids and Structures, 23:211-237, 1987.

44. J. D. Foley, A. van Dam, S. K. Feiner, and J. F. Hughes. Computer Graphics: Principles and Practice. Addison-Wesley Professional, 2nd ed., 1995.

45. Y. Forterre and O. Pouliquen. Flows of Dense Granular Media. Annual Review of Fluid Mechanics, 40:1-24, 2008.

46. D. Freßmann and P. Wriggers. Advection Approaches for Single-and Multi-Material Arbitrary Lagrangian-Eulerian Finite Element Procedures. Computational Mechanics, 39:153-190, 2007.

47. S. Galera, P.-H. Maire, and J. Breil. A Two-Dimensional Unstructured CellCentered Multi-Material ALE Scheme Using VOF Interface Reconstruction. Journal of Computational Physics, 229:5755-5787, 2010.

48. J. D. Goddard. Continuum Modeling of Granular Media. Applied Mechanics Reviews, 66(050801), 2014.

49. M. A. Goodman and S. C. Cowin. A Continuum Theory for Granular Materials. Archive for Rational Mechanics and Analysis, 44:249-266, 1972.

50. G. Greiner and K. Hormann. Effcient Clipping of Arbitrary Polygons. ACM Transactions on Graphics, 17(2):71-83, 1998.

51. G. Gudehus. A Comprehensive Constitutive Equation for Granular Materials. Soils and Foundations, 36(1):1-12, 1996.

52. D. Gueyffier and J. Li and A. Nadim and R. Scardovelli and S. Zaleski. Volumeof-Fluid Interface Tracking with Smoothed Surface Stress Methods for ThreeDimensional Flows. Journal of Computational Physics, 152(2):423-456, 1999.

53. E. Haines. Point in Polygon Strategies. Volume IV of Graphics Gems, pages 24-46. Academic Press, Inc., 1994. 
54. M. Hassanizadeh and W. G. Gray. General Conservation Equations for MultiPhase Systems: 1. Averaging Procedure. Advances in Water Resources, 2:131-144, 1979.

55. M. Hassanizadeh and W. G. Gray. Mechanics and Thermodynamics of Multiphase Flow in Porous Media including Interphase Boundaries. Advances in Water Resources, 13(4):169-186, 1990.

56. U. Häussler and J. Eibl. Numerical Investigations On Discharging Silos. Journal of Engineering Mechanics, 110(6):957-971, 1984.

57. C. W. Hirt, A. A. Amsden, and J. L. Cook. An Arbitrary Lagrangian-Eulerian Computing Method for all Flow Speeds. Journal of Computational Physics, 14:227253, 1974.

58. C. W. Hirt and B. D. Nichols. Volume of Fluid (VOF) Method for the Dynamics of Free Boundaries. Journal of Computational Physics, 39:201-225, 1981.

59. K. Hutter and K. R. Rajagopal. On Flows of Granular Materials. Continuum Mechanics and Thermodynamics, 6:81-139, 1994.

60. K. Hutter, B. Svendsen, and D. Rickenmann. Debris Flow Modeling: A Review. Continuum Mechanics and Thermodynamics, 8:1-35, 1996.

61. H. Hwang and K. Hutter. A New Kinetic Model for Rapid Granular Flow. Continuum Mechanics and Thermodynamics, 7:357-384, 1995.

62. J. M. Hyman. Numerical Methods for Tracking Interfaces. Physica D 12:396-407, 1984.

63. R. M. Iverson. The Physics of Debris Flows. Reviews of Geophysics, 35(3):245-296, 1997.

64. R. M. Iverson and R. P. Denlinger. Flow of Variably Fluidized Granular Masses Across Three-Dimensional Terrain. 1. Coulomb Mixture Theory. Journal of Geophysical Research, 106(B1):537-552, 2001.

65. P. C. Johnson and R. Jackson. Frictional-Collisional Constitutive Relations for Granular Materials, with Application to Plane Shearing. Journal of Fluid Mechanics, 176:67-93, 1987.

66. P. Jop, Y. Forterre, and O. Pouliquen. A Constitutive Law for Dense Granular Flows. Nature, 441:727-730, 2006.

67. D. H. Kim and M.-J. Kim. An Extension of Polygon Clipping to Resolve Degenerate Cases. Computer-Aided Design and Applications, 3(1-4):447-456, 2006.

68. D. Kolymbas. Constitutive Modelling of Granular Materials. Springer-Verlag Berlin Heidelberg, 2000.

69. S. L. Kramer, M. B. Byers, and C. H. Wang. Experimental Measurement of the Residual Strength of Particulate Materials. In P. V. Lade and J. A. Yamamuro (eds): Physics and Mechanics of Soil Liquefaction, pages 249-260. A. A. Balkema, Rotterdam, The Netherlands, 1999.

70. R. J. LeVeque. Finite Volume Methods for Hyperbolic Problems. Cambridge University Press, Cambridge, UK, 3rd ed., 2002.

71. R. W. Lewis and B. A. Schrefler. The Finite Element Method in the Static and Dynamic Deformation and Consolidation of Porous Media. John Wiley \& Sons, Chichester, 2nd ed., 1998.

72. X. S. Li. A Sand Model with State-Dependent Dilatancy. Géotechnique, 52(3):173$186,2002$.

73. J. Locat and H. J. Lee. Submarine Landslides: Advances and Challenges. Canadian Geotechnical Journal, 39:193-212, 2002.

74. G. Luttwak and R. L. Rabie. The Multi Material Arbitrary Lagrangian Eulerian Code MMALE and Its Application to Some Problems of Penetration and Impact. 
Technical Report LA-UR-85-2311, Los Alamos National Laboratory, Los Alamos, New Mexico, USA, 1985.

75. H. U. Mair. Review: Hydrocodes for Structural Response to Underwater Explosions. Shock and Vibration, 6:81-96, 1999.

76. L. E. Malvern. Introduction to the Mechanics of a Continuous Medium. Prentice Hall, Inc., New Jersey, 1969.

77. M. T. Manzari and Y. F. Dafalias. A Critical State Two-Surface Plasticity Model for Sands. Géotechnique, 47(2):255-272, 1997.

78. D. G. Masson, C. B. Harbitz, R. B. Wynn, G. Pedersen, and F. Løvholt. Submarine Landslides: Processes, Triggers and Hazard Prediction. Philosophical Transactions of the Royal Society of London. Series A, 364:2009-2039, 2006.

79. J. M. McGlaun and S. L. Thompson. CTH: A Three-Dimensional Shock Wave Physics Code. International Journal of Impact Engineering, 10:351-360, 1990.

80. G. H. Miller and E. G. Puckett. A High-Order Godunov Method for Multiple Condensed Phases. Journal of Computational Physics, 128:134-164, 1996.

81. H. Y. Ming and X. S. Li. Fully Coupled Analysis of Failure and Remediation of Lower San Fernando Dam. Journal of Geotechnical and Geoenvironmental Engineering, 129(4):336-348, 2003.

82. M. Mooney. The Viscosity of a Concentrated Suspension of Spherical Particles. Journal of Colloid Science, 6(2):162-170, 1951.

83. A. Niemunis and I. Herle. Hypoplastic Model for Cohesionless Soils with Elastic Strain Range. Mechanics of Cohesive-Frictional Materials, 2:279-299, 1997.

84. M. A. Nikolinakou, A. J. Whittle, S. A. Savidis, and U. Schran. Prediction and Interpretation of the Performance of a Deep Excavation in Berlin Sand. Journal of Geotechnical and Geoenvironmental Engineering, 137(11):1047-1061, 2011.

85. J. O'Rourke. Computational Geometry in C. Cambridge University Press, New York, 2nd ed., 1998.

86. S. L. Passman, J. W. Nunziato, P. B. Bailey, and K. W. Reed. Shearing Motion of a Fluid-Saturated Granular Material. Journal of Rheology, 30(1):167-192, 1986.

87. M. Pastor, O. C. Zienkiewicz, and A. H. C. Chan. Generalized Plasticity and the Modelling of Soil Behaviour. International Journal for Numerical and Analytical Methods in Geomechanics, 14:151-190, 1990.

88. J. S. Peery and D. E. Carroll. Multi-Material ALE Methods in Unstructured Grids. Computer Methods in Applied Mechanics and Engineering, 187:591-619, 2000.

89. J. E. Pilliod and E. G. Puckett. Second-Order Accurate Volume-of-Fluid Algorithms for Tracking Material Interfaces. Journal of Computational Physics, 199:465502, 2004.

90. S. P. Pudasaini, Y. Wang, and K. Hutter. Modelling Debris Flows Down General Channels. Natural Hazards and Earth Systems Sciences, 5:799-819, 2005.

91. S. P. Pudasaini. A General Two-Phase Debris Flow Model. Journal of Geophysical Research, 117(F03010), 2012.

92. F. Rackwitz and S. A. Savidis. Numerische Untersuchungen zum Tragverhalten von Zugpfählen in Berliner Sand. Bauingenieur, 79(9):375-383, 2004.

93. W. J. Rider and D. B. Kothe. Reconstructing Volume Tracking. Journal of Computational Physics, 141:112-152, 1998.

94. W. J. Rider, E. Love, M. K. Wong, O. E. Strack, S. V. Petney, and D. A. Labreche. Adaptive Methods for Multi-Material ALE Hydrodynamics. International Journal for Numerical Methods in Fluids, 65:1325-1337, 2011.

95. M. Rudman. Volume-Tracking Methods for Interfacial Flow Calculations. International Journal for Numerical Methods in Fluids, 24(7):671-691, 1997. 
96. S. B. Savage and K. Hutter. The Motion of a Finite Mass of Granular Material Down a Rough Incline. Journal of Fluid Mechanics, 199:21-24, 1989.

97. S. A. Savidis, D. Aubram, and F. Rackwitz. Arbitrary Lagrangian-Eulerian Finite Element Formulation for Geotechnical Construction Processes. Journal of Theoretical and Applied Mechanics, 38(1-2):165-194, 2008.

98. S. A. Savidis, D. Aubram, and F. Rackwitz. Vibro-Injection Pile Installation in Sand: Part II-Numerical and Experimental Investigation. In Th. Triantyfyllidis (ed): Holistic Simulation of Geotechnical Installation Processes - Numerical and Physical Modelling, pages 103-131. Springer International Publishing, 2015. http://dx.doi. org/10.1007/978-3-319-18170-7_6

99. A. Sawicki and J. Mierczyński. Developments in Modeling Liquefaction of Granular Soils, Caused by Cyclic Loads. Applied Mechanics Reviews, 59:91-106, 2006.

100. R. Scardovelli and S. Zaleski. Direct Numerical Simulation of Free-Surface and Interfacial Flow. Annual Review of Fluid Mechanics, 31:567-603, 1999.

101. P. J. Schneider and D. H. Eberly. Geometric Tools for Computer Graphics. Morgan Kaufmann, 2003.

102. A. N. Schofield and C. P. Wroth. Critical State Soil Mechanics. McGraw-Hill, New York, 1968.

103. M. Shashkov. Closure Models for Multimaterial Cells in Arbitrary LagrangianEulerian Hydrocodes. International Journal for Numerical Methods in Fluids 56(8):1497-1504, 2008.

104. H. B. Seed, K. L. Lee, I. M. Idriss, and F. I. Makdisi. The Slides in the San Fernando Dams During the Earthquake of February 9, 1971. Journal of the Geotechnical Engineering Division, ASCE, 101(7):651-688, 1975.

105. R. B. Seed, K. O. Cetin, R. E. S. Moss, A. M. Kammerer, J. Wu, J. M. Pestana, M. F. Riemer, R. B. Sancio, J. D. Bray, R. E. Kayen, and A. Faris. Recent Advances in Soil Liquefaction Engineering: A Unified and Consistent Framework. Technical Report EERC 2003-06, University of California, Berkeley, California, USA, 2003.

106. D. Sunday. Fast Polygon Area and Newell Normal Computation. Journal of Graphics Tools, 7(2):9-13, 2002. http://geomalgorithms.com

107. D. Sunday. Geometry Algorithms: Line and Segment Intersections. http:// geomalgorithms.com/a05-_intersect-1.html, June 2015.

108. I. E. Sutherland and G. W. Hodgman. Reentrant Polygon Clipping. Communications of the ACM, 17(1):32-42, 1974.

109. M. Taiebat and Y. F. Dafalias. SANISAND: Simple Anisotropic Sand Plasticity Model. International Journal for Numerical and Analytical Methods in Geomechanics, 32:915-948, 2008.

110. K. Terzaghi. Theoretical Soil Mechanics. John Wiley \& Sons, Inc., New York, 1943.

111. Th. Triantafyllidis. Cyclic Behaviour of Soils and Liquefaction Phenomena. A.A. Balkema Publishers, The Netherlands, 2004.

112. Th. Triantafyllidis. Holistic Simulation of Geotechnical Installation Processes Numerical and Physical Modelling. Vol. 77 in Lecture Notes in Applied and Computational Mechanics. Springer International Publishing, 2015. http://dx.doi.org/ 10.1007/978-3-319-18170-7

113. C. Truesdell and R. A. Toupin. Encyclopedia of Physics. Bd. III/1: The Classical Field Theories, pages 226-793. Springer-Verlag Berlin Göttingen Heidelberg, 1960.

114. C. Truesdell and W. Noll. The Non-Linear Field Theories of Mechanics. SpringerVerlag Berlin Heidelberg New York, 3rd ed., 2004. 
115. P.-A. von Wolffersdorff. A Hypoplastic Relation for Granular Materials with a Predefined Limit State Surface. Mechanics of Cohesive-Frictional Materials, 1:251271, 1996.

116. Y. Wang and K. Hutter. A Constitutive Model of Multiphase Mixtures and its Application in Shearing Flows of Saturated Solid-Fluid Mixtures. Granular Matter, 1:163-181, 1999.

117. P. Wriggers. Nonlinear Finite Element Methods. Springer-Verlag Berlin Heidelberg, 2008.

118. D. L. Youngs. Time-Dependent Multi-Material Flow with Large Fluid Distortion, pages 273-285. In K. W. Morton und M. J. Baines (eds.): Numerical Methods for Fluid Dynamics. Academic Press, London, 1982.

119. D. L. Youngs. An Interface Tracking Method for a 3D Eulerian Hydrodynamics Code. Technical Report AWRE/44/92/35, Atomic Weapons Research Establishment, Aldermaston, UK, 1987.

120. O. C. Zienkiewicz, A. H. C. Chan, M. Pastor, D. K. Paul, and T. Shiomi. Static and Dynamic Behaviour of Soils: A Rational Approach to Quantitative Solutions. I. Fully Saturated Problems. Proceedings of the Royal Society of London. Series A, 429:285-309, 1990.

121. O. C. Zienkiewicz, A. H. C. Chan, M. Pastor, B. A. Schrefler, and T. Shiomi. Computational Geomechanics - With Special Reference to Earthquake Engineering. John Wiley \& Sons, Chichester, 1999. 